\title{
Review Article \\ Intracellular and Plasma Membrane Events in Cholesterol Transport and Homeostasis
}

\author{
Dmitry Y. Litvinov, Eugeny V. Savushkin, and Alexander D. Dergunov \\ National Research Centre for Preventive Medicine, 10 Petroverigsky Street, 101990 Moscow, Russia \\ Correspondence should be addressed to Alexander D. Dergunov; dergunov@img.ras.ru
}

Received 11 May 2018; Accepted 26 July 2018; Published 6 August 2018

Academic Editor: Xian-Cheng Jiang

Copyright (C) 2018 Dmitry Y. Litvinov et al. This is an open access article distributed under the Creative Commons Attribution License, which permits unrestricted use, distribution, and reproduction in any medium, provided the original work is properly cited.

Cholesterol transport between intracellular compartments proceeds by both energy- and non-energy-dependent processes. Energydependent vesicular traffic partly contributes to cholesterol flux between endoplasmic reticulum, plasma membrane, and endocytic vesicles. Membrane contact sites and lipid transfer proteins are involved in nonvesicular lipid traffic. Only "active" cholesterol molecules outside of cholesterol-rich regions and partially exposed in water phase are able to fast transfer. The dissociation of partially exposed cholesterol molecules in water determines the rate of passive aqueous diffusion of cholesterol out of plasma membrane. ATP hydrolysis with concomitant conformational transition is required to cholesterol efflux by ABCA1 and ABCG1 transporters. Besides, scavenger receptor SR-B1 is involved also in cholesterol efflux by facilitated diffusion via hydrophobic tunnel within the molecule. Direct interaction of ABCA1 with apolipoprotein A-I (apoA-I) or apoA-I binding to high capacity binding sites in plasma membrane is important in cholesterol escape to free apoA-I. ABCG1-mediated efflux to fully lipidated apoA-I within high density lipoprotein particle proceeds more likely through the increase of "active" cholesterol level. Putative cholesterol-binding linear motifs within the structure of all three proteins ABCA1, ABCG1, and SR-B1 are suggested to contribute to the binding and transfer of cholesterol molecules from cytoplasmic to outer leaflets of lipid bilayer. Together, plasma membrane events and intracellular cholesterol metabolism and traffic determine the capacity of the cell for cholesterol efflux.

\section{Introduction}

Cholesterol homeostasis is a well-coordinated machinery of de novo cholesterol synthesis in endoplasmic reticulum and uptake of cholesterol-containing low-density lipoproteins (LDL). Cholesterol synthesis is under tight control by lipoprotein-derived cholesterol that includes inhibition of the sterol regulatory element-binding protein (SREBP) pathway with the decreased expression of genes involved in cholesterol synthesis and uptake [1]. Cholesterol turnover is normally balanced by cholesteryl ester formation at cholesterol excess and cellular cholesterol efflux by both passive and active transport. Reverse cholesterol transport from the cell to the liver is considered as a major atheroprotective event with cholesterol efflux as a rate-limiting step [2,3]. Eukaryotic cells maintain a gradient in sterol concentration between plasma membrane (PM) and the membranes of cell organelles such as endoplasmic reticulum (ER) by both vesicular and nonvesicular mechanisms involving lipid transport proteins.
PM contains $40-90 \%$ of total cellular cholesterol [4], for example, $64 \%$ in $\mathrm{CHO}$ cells [5] and $90 \%$ in human fibroblasts and FU5AH rat hepatoma cells $[6,7]$. ER is the site of cholesterol synthesis; however in contrast to PM, the ER contains just a small fraction of total cell cholesterol, such as $0.5 \%$ of total cell cholesterol in human fibroblasts [8]. Such a drastic difference in cholesterol pools in the PM and ER is due to much higher cholesterol concentration in PM compared to ER and other intracellular membranes. The fraction of cholesterol in PM relative to total PM lipids is $30-40 \mathrm{~mol} \%$ in leukocytes, epithelial cells, neurons, and mesenchymal cell [9]. The sterol/phospholipid ratio in RAW264.7 macrophages and mouse fibroblast LM cells is $0.5-0.6$ for PM, as compared to $0.25-0.3$ for $\mathrm{ER}$, and 0.05-0.1 for mitochondrial membrane $[10,11]$. The ratio of cholesterol to combined cholesterol and phospholipid (PL) content for ER for $\mathrm{CHO}$ cells is $5-7 \mathrm{~mol} \%$, compared to $35 \mathrm{~mol} \%$ for the whole cell $[12,13]$. All other intracellular membranes have smaller cholesterol concentration than the 
PM. Importantly, the PM cholesterol is the cholesterol that participates in the efflux to the extracellular acceptors [14]. Despite the large size of PM cholesterol pool, efflux of just a small fraction of PM cholesterol can be significantly affected by intracellular events. Indeed, while cholesterol efflux in cholesterol-depleted RAW 264.7 cells is as small as less than $1 \%$ of total cholesterol, the knockdown of dynamin-2, or ADP-ribosylation factor 6 (Arf6), or Cdc42 reduces it even further [15]. The efflux of $2 \%$ of total cell cholesterol in THP1 cells was almost completely inhibited by the knockdown of oxysterol-binding protein-related protein 6 (OSBPL6, aka ORP6) [1]. The efflux of $4 \%$ of total cholesterol in peritoneal macrophages from WT mouse was reduced by 2 -fold in the peritoneal macrophages with knockout in Niemann-Pick disease, type C1 (NPC1) protein [16]. Another illustration of the effect of intracellular or PM processes on cholesterol efflux is the overexpression of caveolin-1 in RAW 264.7 that increases cholesterol efflux to HDL from $2.5 \%$ to almost 5\% [17]. The lipid composition and structural features of plasma membrane $[18,19]$ also contribute to cholesterol efflux.

The goal of this review is to describe the complex processes of cholesterol metabolism and cholesterol traffic inside the cell and the effect of these processes on the cholesterol efflux from the cells. The mechanisms of cholesterol transfer between cell membranes and underlying reason of gradient of cholesterol concentration between intracellular and plasma membranes will be discussed. We also describe four known mechanisms of cholesterol efflux-aqueous diffusion, facilitated diffusion mediated by SR-B1 receptor, and active unidirectional efflux mediated by ABCA1 and ABCG1 transporters. The contribution of different pools of cholesterol and types of acceptor will be also considered.

\section{Lipid Rafts and Cholesterol Pools in Lipid Bilayer and Cell Membranes}

2.1. Membrane Lipid Composition and Two Kinetic Pools of PM Cholesterol. The distribution of lipids between membrane leaflets is not even. The cytosolic leaflet of PM is enriched with PE, PI, and PS compared to outward leaflet and is poorer in sphingolipids and PC $[10,20]$. In addition, the composition of the membranes is not isotropic because of rafts and other structural features [21]. Dependent on the membrane composition, cholesterol can be associated with other lipids in more or less tight and stable complexes that affect its activity. The ability to form a complex and its stoichiometry depends on the lipid structure, such as the saturation and length of the acyl chains and the size of the polar head. The lipid:cholesterol molar ratio in the complexes usually varies between 3:1 and 1:1. Cholesterol preferably interacts with lipids containing large polar heads and saturated fatty acid residues due to increased shielding of cholesterol from the aqueous phase by large heads and a stronger Van der Waals interaction with saturated chains [18]. Under certain conditions, the addition of cholesterol to phospholipids leads to phase separation: phosphatidylcholine and especially sphingomyelin concentrate in the ordered and condensed cholesterol-rich phase, while the remaining phospholipids are displaced into the liquid phase. The pressure-composition phase diagram

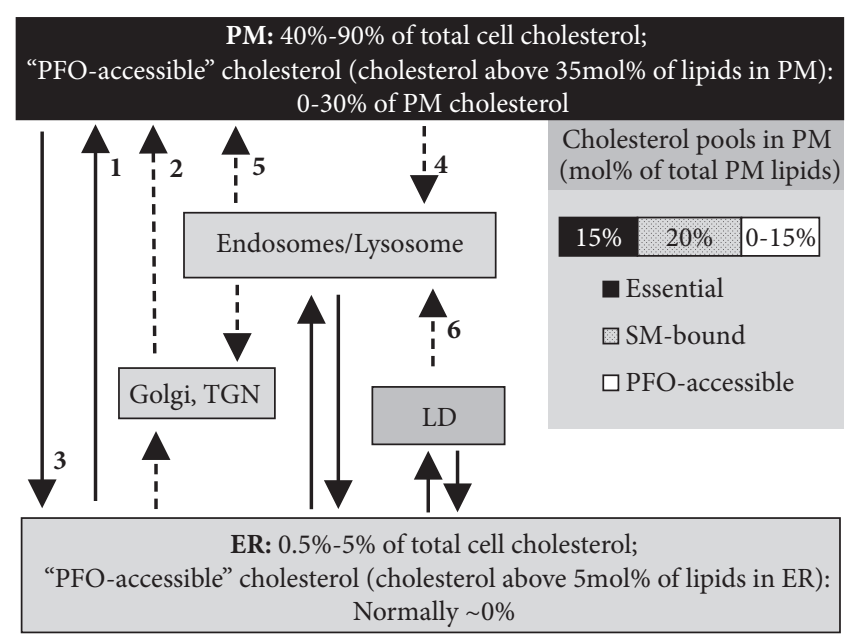

FIgURE 1: Cholesterol distribution and movement between major compartments. Solid arrows indicate nonvesicular cholesterol transport (including transport via membrane contact sites and lipid transfer proteins); dashed arrows indicate vesicular transport and transport of cholesterol mediated by organelles. The numbers indicate the following: (1) the major nonvesicular, not cytoskeletondependent, energy-dependent path of cholesterol transport from ER to PM; (2) the minor vesicular transport from ER to PM via Golgi; (3) not energy-dependent, cytoskeleton-dependent cholesterol transport from PM to ER; (4) transport of LDL bound by LDL receptor in clathrin-coated vesicles; (5) energy-dependent, cytoskeleton-dependent cholesterol transport via lysosome-related organelles; (6) lysosomal degradation of LD. Three pools of cholesterols with different accessibility to water phase are known in PM. PFO-accessible cholesterol is the most available pool for the interactions with reagents in aqueous phase, such as PFO (Perfringolysin O), cholesterol oxidase, and cyclodextrin. This pool with variable size is considered as a putative "active" cholesterol. The size of this pool is very small at cholesterol depletion, while at the increase of its size in PM cholesterol moves to the ER. PFO-accessible pool in ER appears at much smaller mol\% of cholesterol. PM (plasma membrane), ER (endoplasmic reticulum), LD (lipid droplets), TGN (trans-Golgi network), LDL (low-density lipoprotein), and SM (sphingomyelin).

for cholesterol-phospholipid mixtures is characterized by an initial increase in pressure at low cholesterol content caused by the formation of ordered regions in the liquid phase. As cholesterol content increases, the pressure decreases due to the formation of a stoichiometric complex. The pressure then increases again when the liquid regions are formed in the condensed phase with a further increase in the cholesterol content [19]. Cholesterol, not associated in complexes with other lipids, is more available for reactions and more active at the escape from the membrane, probably due to disruption of its orientation in the bilayer. Cholesterol molecules with higher chemical activity are more accessible for the removal by cholesterol acceptors, for oxidation by cholesterol oxidase, and for binding to bacterial protein toxins of cholesteroldependent cytolysins family, e.g., Perfringolysin O (PFO) and anthrolysin $[19,22,23]$. In the membrane, cholesterol pools with different activity can simultaneously be present (Figure 1). It was suggested that cholesterol distribution in 
membranes of various organelles depends on the composition of these membranes and corresponds to the stoichiometry of the cholesterol complexes. Two existing views on uneven cholesterol content in plasma and intracellular membranes suggest (1) the distribution of cholesterol by diffusion or assisted diffusion of cholesterol, which depends on cholesterol concentration and lipid composition of the membranes, and (2) the participation of energy-dependent active transport of cholesterol by cholesterol transfer proteins $[19,24]$. Cholesterol level in endoplasmic reticulum does not exceed 5\% and there is no complex with 19:1 stoichiometry; the complex formation is discarded for such phospholipid as 1-stearoyl-2-oleoyl-sn-glycero-3-phosphatidylcholine. Alternatively, the diminished cholesterol content may originate from the presence in ER of any substances that exclude cholesterol from the complex.

Cholesterol level is tightly maintained in cell membrane due to complex formation. Thus, the excess of cholesterol in the PM results in the cholesterol efflux or its transfer to ER for conversion to $\mathrm{CE}$ or in some cases to mitochondria for oxidation to oxysterols [25]. Normally, the cholesterol content of PM of fibroblasts and $\mathrm{CHO}$ cells is about $40-50 \%$ of total PM lipids. The association of plasma membrane cholesterol with a mutant form of bacterial Perfringolysin $\mathrm{O}(\mathrm{PFO} *)$ occurs at cholesterol increase over $35 \%$. Thus up to $~ 15 \%$ are available for the binding to the $\mathrm{PFO} *$. This cholesterol pool disappeared when cells are cholesterol-depleted. The treatment of the cells with sphingomyelinase releases cholesterol from cholesterol-SM complexes thus adding $10-23 \%$ of the cholesterol to the $\mathrm{PFO} *$-accessible pool. The rest of the cholesterol does not bind to $\mathrm{PFO} *$. It is an essential pool, and the depletion of this cholesterol results in the rounding of the cells and their detachment to the medium. The binding of ER cholesterol with PFO occurs at cholesterol level over 5\%. The relation between static and kinetic cholesterol pools remains undetermined; however, the concentration of cholesterol complex with sphingomyelin decreased at the increase of cyclodextrin content $[14,26]$.

Two pools of cholesterol are observed in the kinetics of cholesterol efflux to cyclodextrin in Fu5AH hepatoma cells, mouse fibroblasts L-cells, human skin fibroblasts, and CHO$\mathrm{K} 1$ cells. The fast pool is $20-60 \%$ (the highest is for the Fu5AH cells) of cell cholesterol and the efflux half time is about 15$23 \mathrm{sec}$. The slow pool is $50-80 \%$ and its half time of efflux is about $15-35$ min $[14,27]$. The cholesterol efflux to $\mathrm{HDL}_{3}$ in $\mathrm{CHO}-\mathrm{K} 1$ cells similarly to the efflux to cyclodextrin shows fast and slow pools. However, the sizes of both pools for the efflux to HDL3 are much smaller than if the cholesterol acceptor is cyclodextrin. The temperature dependence of cholesterol efflux indicates that activation energy for cholesterol transfer to $\mathrm{HDL}_{3}$-derived apoHDL-PC is $20 \mathrm{kcal} / \mathrm{mol}$, which is much higher than for cyclodextrin $(7 \mathrm{kcal} / \mathrm{mol})$. Slow and fast pools exchange cholesterol with a half time 20-30 min. All cholesterol molecules of the fast pool and the majority of cholesterol of the slow pool escape from the PM [14, 27].

Energy poisons do not significantly affect the fast pool of cholesterol. Sodium azide $\left(\mathrm{NaN}_{3}\right)$, potassium cyanide (KCN), sodium fluoride $(\mathrm{NaF})$, ATPase inhibitor bafilomycin $\mathrm{Al}$, or mixture of $\mathrm{NaN}_{3}$ with 2-deoxyglucose does not affect half-times and size of fast cholesterol pool in CHO-K1 cells or just slightly affects them in $\mathrm{CHO}$ cells expressing human transferrin receptor $[14,28]$. The data on the effect of energy poisons on the parameters of slow cholesterol pool are controversial. $\mathrm{NaN}_{3}, \mathrm{KCN}, \mathrm{NaF}$, and ATPase inhibitor bafilomycin A1 do not affect half time and size of slow cholesterol pool in CHO-K1 cells [14]. However, energy poison mixture of $\mathrm{NaN}_{3}$ with 2-deoxyglucose effectively prevents cholesterol efflux from the slow pool in $\mathrm{CHO}$ cells expressing human transferrin receptor [28].

\subsection{Cholesterol Homeostasis Might Be Regulated by "Active"} Cholesterol. The difference in the chemical activity of cholesterol between various membranes results in the cholesterol flux from one membrane to another [22]. Sphingomyelins (SMs) are known as lipids that can be associated with cholesterol in membranes. Partial hydrolysis of SMs by treatment of the cells with sphingomyelinase results in rapid flux of cholesterol from PM to intracellular cholesterol pools [72]. It can be assumed that many processes that depend on individual cholesterol molecules, such as cholesterol diffusion and cholesterol-protein interaction, similarly depend on the concentration of the accessible "active" cholesterol, but not the total cholesterol. An increase of ER cholesterol above $\sim 5 \mathrm{~mol} \%$ results in the appearance of PFO-accessible cholesterol and in the disappearance of SREBP-2 in the nucleus controlled by SREBP cleavage-activating protein (SCAP). It suggests that SCAP protein senses the "active" cholesterol in the membrane and becomes activated in the presence of active cholesterol [12]. On the contrary, the "active" cholesterol inhibits the activity of ER-associated enzyme HMG-CoA reductase. Its activity is minimal at normal or increased "active" cholesterol in PM but increases when the cholesterol level is not enough for the appearance of the active cholesterol [73].

A model was proposed that explains sterol gradient between ER and PM and the ability of the sterol-poor ER to respond to the small changes in sterol content in the sterol-rich PM. It suggests that PM rafts contain the most of PM sterols, which is not "free" (or "active") and does not participate in the exchange with ER cholesterol. "Free" (not raft-associated) cholesterol is transferred between ER and PM by nonvesicular mechanism. The concentration of "free" cholesterol in the ER and PM is about the same, and the difference in the total cholesterol content between ER and $\mathrm{PM}$ is caused by the removal of the active cholesterol into the lipid rafts [74]. The concentration of "active" cholesterol depends on the cholesterol concentration and the membrane composition [75]. Indeed, while PFO-sensitive pool of cholesterol in the ER appears at about $5 \mathrm{~mol} \%$ of cholesterol, the PM needs around $35 \mathrm{~mol} \%$ cholesterol before it starts to appear in PFO-sensitive pool [76]. Thus, the gradient of cholesterol concentration between the PM and ER might be thermodynamically stable.

\section{Intracellular Cholesterol Turnover}

A number of intracellular proteins affect cholesterol efflux to extracellular acceptors of cholesterol (Table 1) with activator 


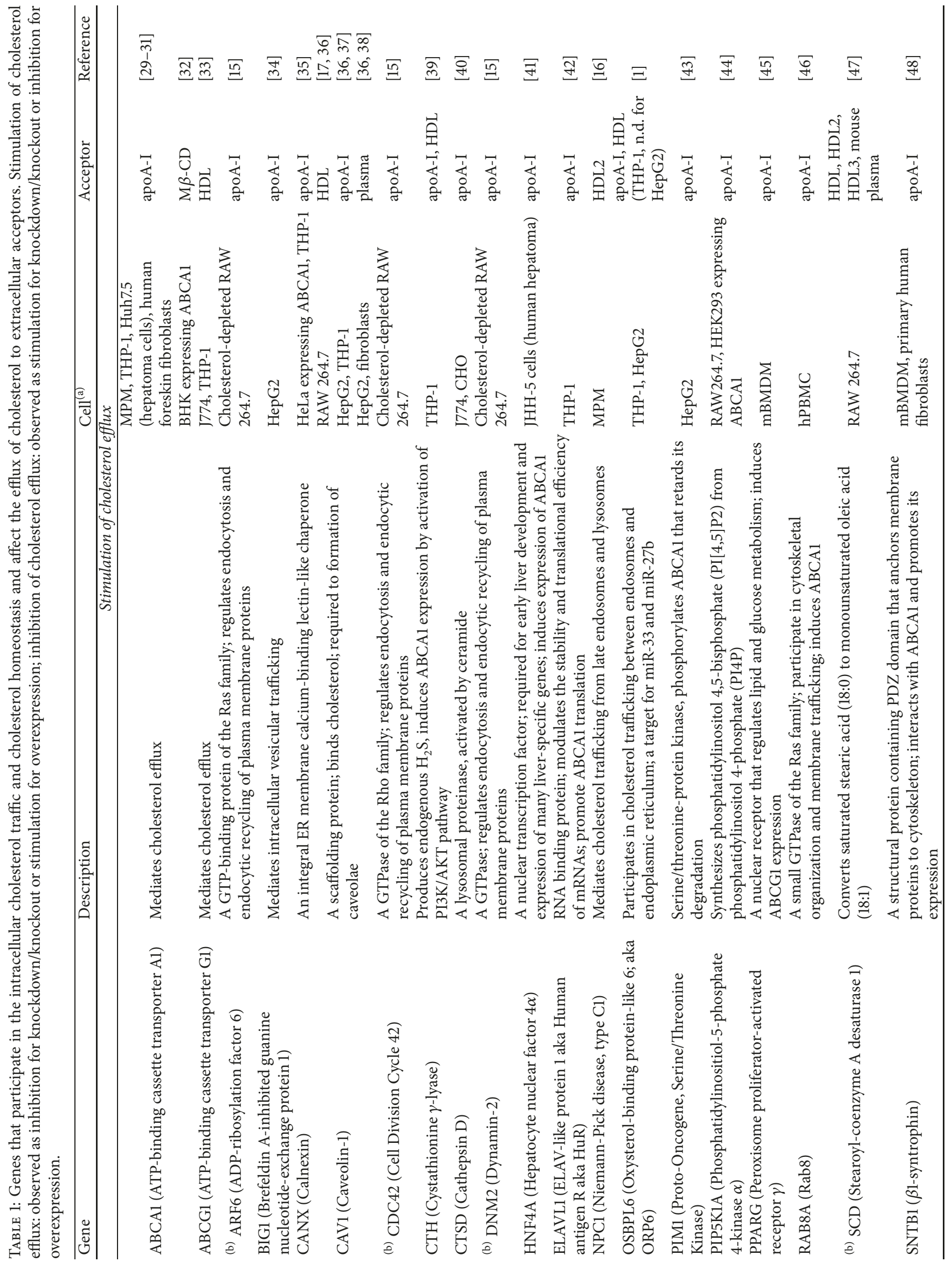




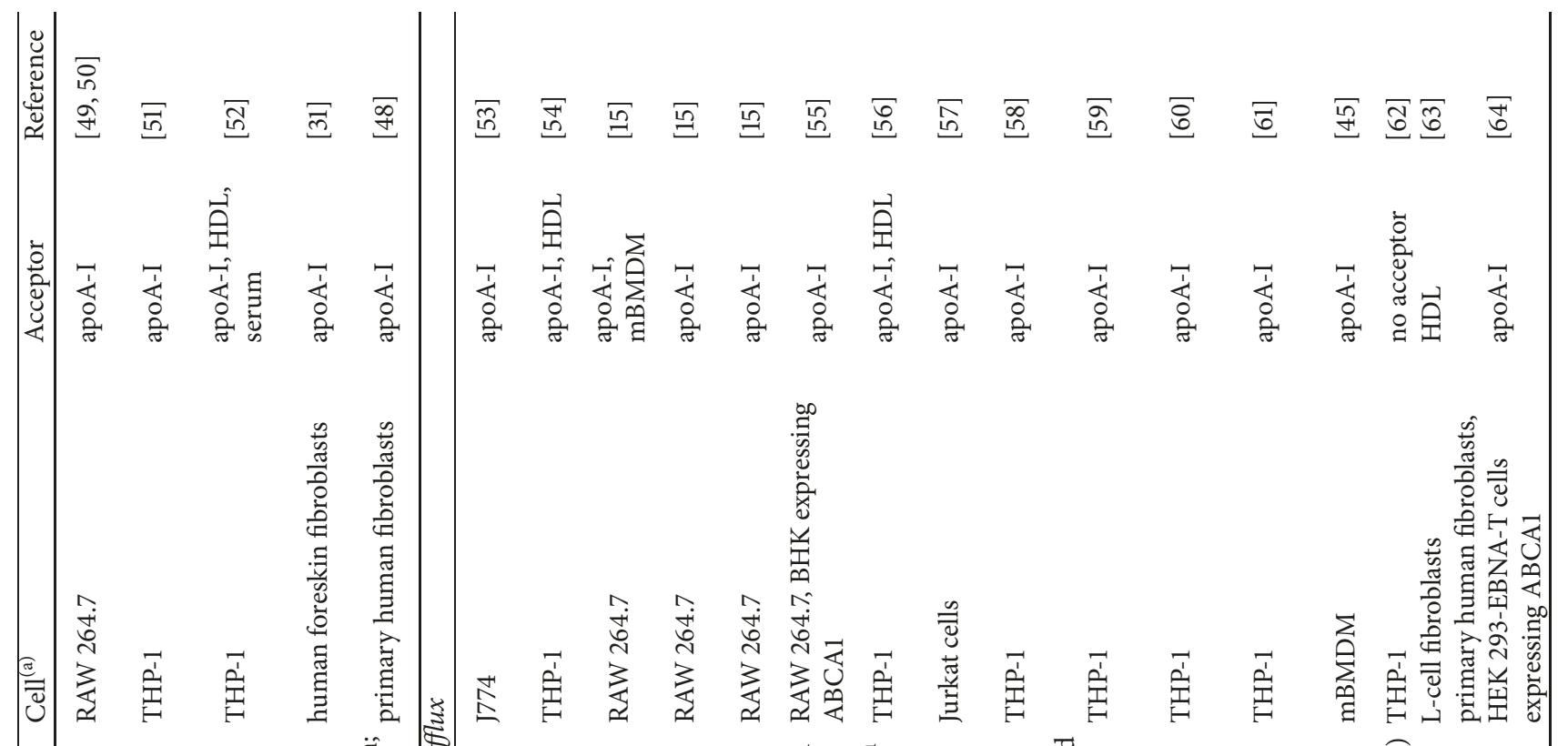

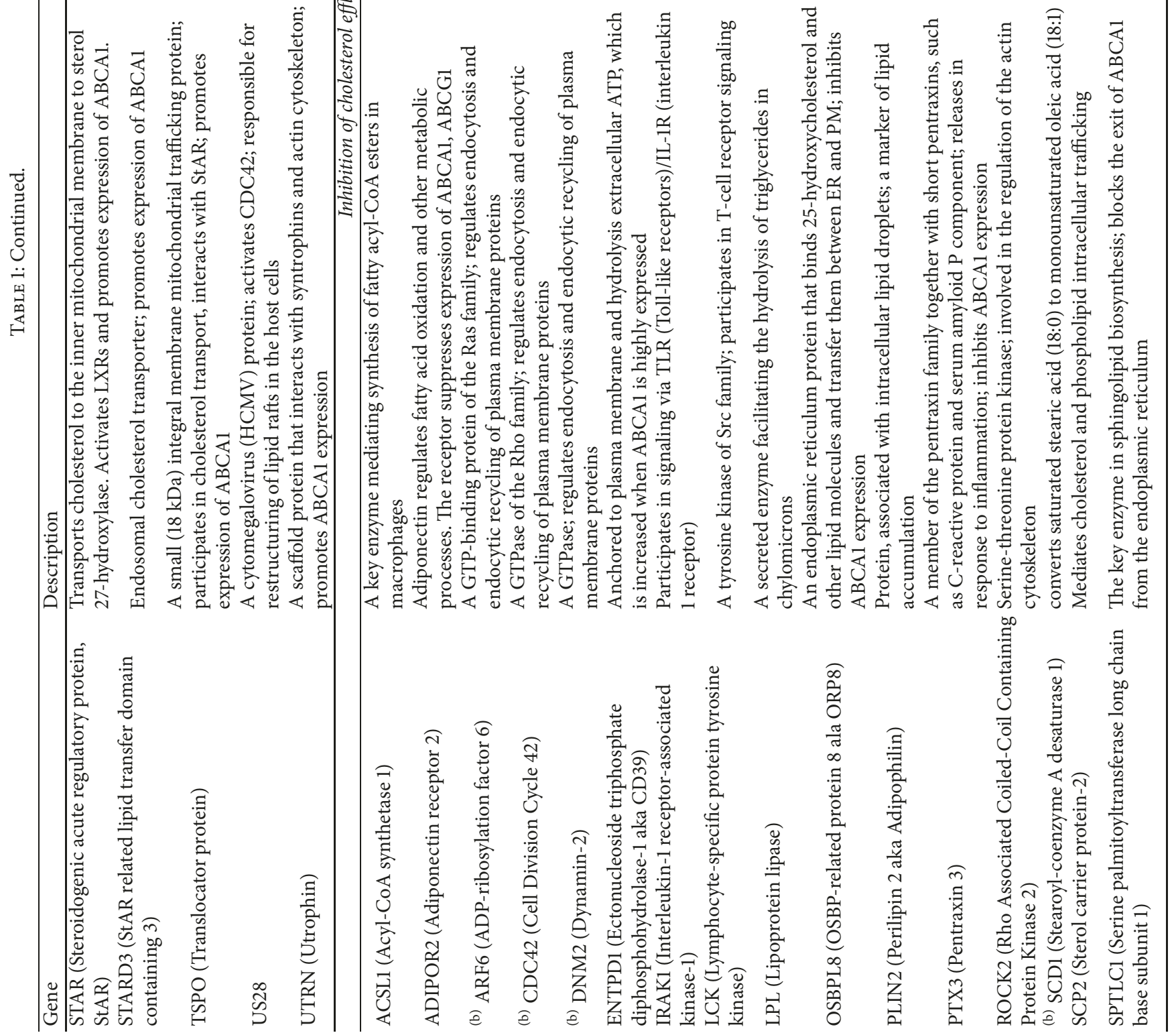




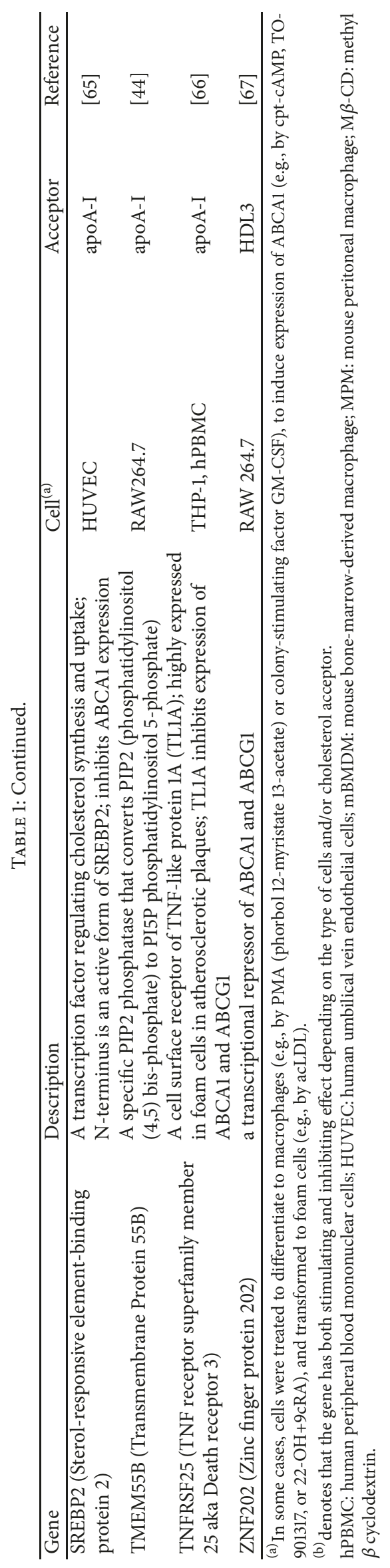


and inhibitor properties toward cholesterol synthesis and uptake, cholesterol distribution in the membrane, intracellular vesicular and nonvesicular trafficking, cytoskeletal organization, and cholesterol escape. It can be assumed that proper cholesterol homeostasis and fully functional intracellular cholesterol transport are required for normal level of cholesterol efflux. The central processes that maintain cholesterol balance and are common for the most of the cells are described below.

3.1. Abundance of Cholesterol Pools. A person every day receives about $400 \mathrm{mg}$ of cholesterol with food while secreting through the liver approximately $1 \mathrm{~g}$ [77]. The rate of cholesterol synthesis in humans is estimated at about $10 \mathrm{mg} /$ day per $\mathrm{kg}$ of body weight. It is assumed that liver contributes roughly $10 \%$ of this rate and the rest of the synthesis occurs in intestine and peripheral tissues [78, 79]. Endogenous cholesterol as well as the majority of other lipids is synthesized by the ER. In particular, rate-limiting enzyme in cholesterol synthesis 3-hydroxy-3-methylglutaryl coenzyme A reductase (HMGCR) is located in the ER. Most cells also uptake cholesterol from lipoproteins in normal conditions [80]. Low-density lipoproteins are internalized by LDL receptor (LDLR) in clathrin-coated vesicles. The vesicle is transported to sorting endosome, where LDL dissociates from the LDL receptor and the latter is recycled back to the plasma membrane via the endocytic recycling compartment (ERC). LDL goes to late endosome (LE), where cholesteryl ester (CE) of LDL is hydrolyzed by acid lipase and resulting cholesterol is distributed mainly to PM, to ER to a lesser degree, and to cell membranes of other organelles [81, 82]. Niemann-Pick type C proteins NPC1 and NPC2 are critically important for unloading of cholesterol from LE. A small GTPase Rab8 participates in cholesterol transport from LE to PM in Myosin5-dependent movement of cholesterolenriched lysosome-related organelles along actin cytoskeleton [82]. Depletion of Rab8 in foam cell inhibits cholesterol efflux to apoA-I in part by the reduction of ABCA1 level at the PM [46]. One of the routes of cholesterol transport from LE to ER is vesicular transport through trans-Golgi network (TGN) and requires NPC1 and v-SNARE vesicle-associated membrane protein 4 . Peritoneal macrophages from NPC1 knockout mouse show less efficient cholesterol efflux compared to the normal peritoneal macrophages (Table 1). In addition, there are membrane contact sites (MCSs) between LE and ER with cholesterol-binding proteins ORP1L, ORP5, and STARD3 that can participate in cholesterol transfer [82]. While cholesterol content of ER is much lower than of PM, it plays essential role in maintaining cholesterol homeostasis as a site of sensing cholesterol level that regulates expression of LDLR and HMGCR and a site of cholesterol synthesis if required and esterification for storage when cholesterol is in excess. Loading of fibroblasts with cholesterol using hydroxypropyl-beta-cyclodextrin-cholesterol complex that increases total cell cholesterol by $50 \%$ results in 10 -fold increase in cholesterol level in the ER from $0.5 \%$ to $5 \%$ of total cell cholesterol. A depletion of the cells by $25 \%$ decreased ER cholesterol by $75 \%$ of original level [8]. An excess of some intermediates of cholesterol synthesis results in HMGCR degradation. Enzyme structure has sterol-sensing domain (SSD) and it is subjected to proteasome degradation if it binds oxysterols, lanosterol, 4,25-dihydrolanosterol but not cholesterol [80]. On the contrary, acyl-coenzyme A:cholesterol acyltransferase (ACAT) is activated by cholesterol and at higher cholesterol level converts it to CE for storage [83].

Both the biosynthesis and uptake of cholesterol are transcriptionally regulated by sterol regulatory element-binding protein family of SREBP-1a, SREBP-1c, and SREBP-2. The function of SREBPs is dependent on proper protein trafficking from ER to Golgi. When cell does not require a supply of cholesterol, SREBPs are anchored to ER. At low cholesterol condition, sterol-sensing domain (SSD) of SREBP cleavageactivating protein (SCAP) loses cholesterol, and SCAP initiates COPII-mediated incorporation of SREBPs into budding vesicles and transport them from the ER to the Golgi, where SREBPs are cleaved by Site- 1 and Site- 2 proteases. N-termini of SREBPs release into cytoplasm and move to nucleus escorted by importin-beta, where they induce expression of LDLR, HMGCR, Insulin induced gene 1 (Insig-1), and other SREBP target genes. In addition, SREBPs suppress expression of $A B C A 1$ that removes cholesterol to extracellular acceptors. When cholesterol level becomes too high, SCAP/SREBP complex binds to ER-anchored Insig-1, which retains the complex in the ER and prevents induction of target genes [80].

When cells become depleted of cholesterol, they first start to utilize CE stored in lipid droplets (LD) instead of the synthesis of new cholesterol [80]. A large number of CErich LDs is an indicator of macrophage transformation to foam cell because of excessive uptake of cholesterol. Lipid droplets are organelles that store sterol esters, triglycerides, and some other neutral lipids. The neutral lipid core is surrounded by a monolayer of phospholipids that contains a number of proteins that participate in LD metabolism. LDs are formed in ER because of synthesis of neutral lipids, such as $\mathrm{CE}$ that is synthesized from newly synthesized or LDLderived cholesterol and fatty acyl-CoA by ACAT-1 or sterol $\mathrm{O}$-acyltransferase 1 . As the concentration of neutral lipids increases, they cannot be dissolved anymore in the ER membrane, and supposedly, a "lens" of neutral lipids between ER membrane leaflets appears that grows to a "drop" being still attached to the ER membrane. An integral membrane protein Seipin is detected in the LD-ER contact site. It is not fully clear whether fully formed LD stays attached to the ER or separated from the ER. Nevertheless the proteins of COPI vesicle coats are found on the LD surface. COPI vesicle transports cargo from Golgi to ER [84-86]. In macrophage foam cells cholesterol is constantly esterified by ACAT and deesterified by neutral CE hydrolases, such as carboxylesterase 1 and, possibly, neutral cholesterol ester hydrolase 1 [87]. That "futile cycle" is a LD-based mechanism that can help to maintain the normal cholesterol concentration. Beside the cytoplasmic lipolysis, LD can release cholesterol by LD lysosomal degradation in an autophagy route when CE is hydrolyzed by lysosomal acid lipase. In foam cells, the LD incorporates into autophagosome that fuses with lysosome and releases cholesterol for efflux from the cells. Inhibition of autophagy by Atg5 gene knockout reduces cholesterol 
efflux to apoA-I and reverses cholesterol transport to the liver in vivo, and $\mathrm{mTOR}$ inhibitors that stimulate autophagy are atheroprotective $[88,89]$. An inhibition or knockdown of ACAT-1 results in the increase of cholesterol:phospholipid ratio in PM rafts and in the stimulation of ABCA1-dependent cholesterol efflux [90]. Incubation of the cells with cholesterol acceptors depletes cellular CE content [91].

3.2. Vesicular and Nonvesicular Cholesterol Traffic. Intracellular vesicular movement includes lipid transfer from one compartment to another as a constituent of the membrane of secretory vesicles [92, 93]. Anterograde COPII (coat protein complex II) vesicles traverse from the ER to Golgi, and retrograde COPI vesicles traverse from Golgi to the ER. Golgi along with trans-Golgi network is structurally highly dynamic organelle and intra-Golgi vesicle transport is not clearly understood. The Golgi routes the vesicle to $\mathrm{PM}$ and endosomal compartments. Intracellular traffic between compartments depends on various mechanisms. These mechanisms can be roughly described as energydependent and cytoskeleton-dependent. For instance, vesicle transport requires both energy and functional cytoskeleton. Despite the hypothetical possibility of vesicular traffic to transport significant amount of cholesterol, this route plays just a minor role in the cholesterol transfer from the site of the synthesis (ER) to PM. The disruption of cytoskeleton has no effect on the cholesterol transfer from ER to PM, and disruption of vesicle traffic by brefeldin A decreases nascent cholesterol transport to PM just by $20 \%$ [94]. Newly synthesized cholesterol bypasses the esterification to CE and appears in PM with half time of about $15 \mathrm{~min}$ [7]. This transport is inhibited by energy poisons $\mathrm{KCN}+\mathrm{KF}$ or $\mathrm{NaN}_{3}$ $+\mathrm{NaF}$ in both mammalian and yeast cells [74, 95]. The transport of nascent cholesterol to PM stops if the cells are cooled to $15^{\circ} \mathrm{C}$. At this temperature, the nascent cholesterol accumulates in the ER, and after retuning cells to $37^{\circ} \mathrm{C}$ cholesterol transport to PM is restored [95]. The disruption of cytoskeleton by cytochalasin, colchicine, and nocodazole or the disruption of Golgi apparatus by monensin and brefeldin A just slightly inhibits cholesterol transport from ER to PM $[4,95]$. Microtubule disruption reagent nocodazole inhibits the transport just by $25 \%$ and the disruption of Golgi by brefeldin A by $20 \%$ [96].

The PM cholesterol is constantly transported back to ER by not energy-dependent mechanism. This transport is inhibited by disruption of the cytoskeleton and acidic compartments [4]. It was estimated that if the whole PM pool of cholesterol participates in the cycling, then the half time of cholesterol cycling is $40 \mathrm{~min}$ [7]. Considering the hypothesis of some "active" cholesterol in membranes, the half time is even faster. Unlike creating a cholesterol gradient between PM and ER, its maintaining does not depend on energy. Incubation of the cells with energy poisons does not change the distribution of cholesterol between PM and ER [95, 97]. The rate of dehydroergosterol transport to cholesterolenriched endocytic recycling compartment from the PM was not greatly affected by ATP depletion with energy poison mixture of $\mathrm{NaN}_{3}$ with 2-deoxyglucose, indicating that the transport was mainly nonvesicular [28]. Oppositely, the efflux of sterol from ERC to the PM is inhibited significantly by the energy poisons [28] indicating that vesicular transport out of, but not into, the ERC is a major contributor to sterol transport kinetics. However, recent data of the same group suggest rather different mechanism of bidirectional sterol movement. The poison mixture decreased the rate of PM to ERC and ERC to PM transfer by $\sim 30 \%$, so that $\sim 70 \%$ of cholesterol flux is not dependent on vesicle transport in osteosarcoma U20S cells that stably express the scavenger receptor A SRA; the half time of dehydroergosterol transfer between PM and ERC is about $15 \mathrm{~min}$ in both directions [98]. In yeast, the disruption of Sec18p, the yeast orthologue of mammalian Nethylmaleimide sensitive fusion protein, an essential protein for vesicular trafficking between ER, Golgi, and PM, does not inhibit the ER to PM transport [74]; two plasma membrane ABCG transporters, Aus1p and Pdrllp, stimulate cholesterol transport from PM to ER [99].

Membranes of virtually any organelle are interconnected through membrane contact sites (MCSs). MCSs connect ER with PM, Golgi, endosomes, lysosomes, mitochondria, and peroxisomes. The MCSs are organized as protein complexes that link the membranes and hold them in the distance about 10-50nm apart and serve as sites for lipid transfer protein(LTP-) assisted nonvesicle lipid transport. Several LTPs are known to transfer cholesterol between the organelles. The most studied proteins are proteins of ORP family (oxysterolbinding protein- (OSBP-) related proteins) [100-102], STARD family (StAR related lipid transfer domain containing proteins) $[100,103,104]$, and sterol carrier protein 2 (SCP-2) [105].

3.3. Lipid Transfer Proteins. The oxysterol-binding protein (OSBP) is the founder member of the ORP (OSBP-related protein) family that consists of 12 genes in humans and 7 genes in yeast, Osh1-Osh7. All ORP genes contain lipid binding domain [100]. OSBP is predominately cytosolic protein with minor fraction bound to ER. The ER-bound OSBP forms the MCS between ER and Golgi through binding with vesicleassociated membrane protein-associated protein A (VAP-A) [106]. OSBP binds with high affinity to 25-hydroxycholesterol $\left(K_{d}=5 \mathrm{nM}\right)$ and to a number of other oxysterols $[100,107]$. The binding to oxysterols leads to translocation of OSBP as well as VAP-A to perinuclear compartments of the cells [106]. OSBP also binds to cholesterol $\left(\mathrm{K}_{\mathrm{d}}=70 \mathrm{nM}\right)$ and phosphatidylinositol-4-phosphate (PI4P) [100]. Overexpression of OSBP in HeLa cells suppresses sterols incorporation into lipid droplets, while the mutant OSBP protein, which is assumed to lose PI4P binding ability, does not interfere with sterols accumulation in LDs. In vivo and in vitro experiments suggest that OSBP is a PI4P-dependent cholesterol transporter. According to the proposed model, ER-anchored VAP-A at the ER-trans-Golgi MCS binds OSBP, which starts to transfer cholesterol from ER to trans-Golgi, and PI4P in the opposite direction. The gradient of PI4P between two membranes is required, and ER-anchored PI4P phosphatase Sacl helps in maintaining the PI4P gradient by PI4P degradation while supposedly PI4-kinases continuously regenerate PI4P in the Golgi [106]. Thus, this mechanism requires ATP to maintain PI4P gradient. Similarly, Osh4p 
transfers ergosterol between ER and trans-Golgi in yeast. Other ORP/Osh proteins are located in the MCSs between ER and PM and other organelles, and most of them are involved in cholesterol transfer $[101,102]$. A contribution of ORP6 was investigated in THP-1 macrophages and HepG2 hepatocytes. ORP6 localizes in early endosomes, lysosomes, and the endoplasmic reticulum. Loading of THP-1 cells with cholesterol by acetylated low-density lipoprotein (acLDL) induces ORP6 expression. Knockdown of ORP6 in THP1 macrophages upregulates mRNA of SREBF2 and genes induced by $S R E B F 1 / 2, H M G C R$, and $L D L R$. At the same time ORP6 knockdown inhibits cholesterol efflux to apoAI and HDL in THP-1 macrophages and inhibits cholesterol efflux to apoA-I in HepG2 hepatocytes. The overexpression of ORP6 results in the opposite effect [1]. Thus, ORP6dependent cholesterol supply from the intracellular sources significantly affects cholesterol efflux. Knockdown of another ORP protein, ORP8 (or OSBP-related protein 8), in THP1 macrophages stimulates cholesterol efflux to apoA-I in parallel to an increase of ABCA1 protein [59].

StAR (or STARD1) is the founder protein of the STARD family. It transfers cholesterol from the outer mitochondrial membrane to the inner mitochondrial membrane in steroidogenic tissues for hormone synthesis. The rate of the transfer was estimated as 400 molecules of cholesterol/min per molecule of newly synthesized StAR [108]. StAR overexpression stimulates cholesterol efflux by activating LXR and stimulation of ABCA1 expression. The stimulation of the efflux in cAMP-activated RAW 264.7 macrophages is blocked by sterol 27-hydroxylase inhibitor GW273297x, LXR inhibitor geranylgeranyl pyrophosphate, and ABCA1 inhibitor probucol [49]. Overexpression of StAR stimulates cholesterol efflux to apoA-I in RAW 264.7 macrophages stimulated by agonists of retinoic acid receptor and/or retinoid $\mathrm{X}$ receptor alltrans retinoic acid (RA) or 9-cis RA, which activate LXR pathway [50]. STARD3 protein is anchored to late endosomes and together with ER-anchored vesicle-associated membrane protein-associated proteins A and B STARD3 contributes to formation of MCSs between endosomes and ER. Overexpression of STARD3 in HeLa cells, which express STARD3 at very low level, promotes accumulation of cholesterol in LE, while not changing the total cell cholesterol level. Cholesterol depletion of the cells does not prevent cholesterol accumulation in endosomes, while inhibitor of cholesterol synthesis mevinolin prevents the endosomal cholesterol accumulation that indicates on the ER-synthesized cholesterol transport by STARD3. All together, it suggests that STARD3 mediates cholesterol traffic from ER to endosomes, and this route competes with ER to PM traffic. The mechanism of the transfer does not depend on energy [109]. Overexpression of STARD3 in THP-1 cells stimulates cholesterol efflux to apoA-I; however the effect is likely based on the upregulation of ABCA1 mRNA and protein; the efflux to HDL does not change in the STARD3-transfected cells significantly [51]. Another member of STARD family, STARD4 protein, mediates nonvesicular sterol transfer between PM and ERC $[98,110]$. In HepG2 hepatocytes expression of STARD4 is induced when cells are incubated in cholesterol-poor conditions. The overall effect of knockdown of the STARD4 on the cell cholesterol level and intracellular distribution depends on the cell type. The knockdown of the STARD4 in HepG2 cells results in the reduction of cholesterol in endoplasmic reticulum and the reduction in the level of cholesteryl esters without significant changes in total cholesterol level [111]. On the contrary, the knockdown of STARD4 in osteosarcoma cells U20S results in an increase in cholesterol level in PM and ERC and in an increase in the level of cholesteryl esters [110].

Sterol carrier protein 2 (SCP-2) binds cholesterol and phospholipids with high affinity. It is localized in peroxisomes and in cytoplasm and involved in cholesterol and phospholipid intracellular transfer. SCP-x, a longer form of SCP-2 that is transcribed from an alternate transcription site, is a peroxisomal 3-ketoacyl-CoA thiolase [105]. The study of SCP-2-deficient fibroblasts from patients with Zellweger syndrome revealed that roughly $50 \%$ of ER to PM transport of newly synthesized cholesterol is cytoskeleton- and Golgidependent, in contrast to the transport in normal fibroblasts, which does not depend on the cytoskeleton and Golgi. Knockdown of the SCP-2 in normal fibroblasts decreases the fast $(10 \mathrm{~min})$ transport from ER to PM by $80 \%$; however the cholesterol is still able to flux to the PM by slower mechanism [112]. Overexpression of SCP-2 in McA-RH7777 rat hepatoma cells sharply increases the rate of the transfer of newly synthesized cholesterol from ER to PM and the amount of newly synthesized cholesterol in the secreted HDL. The overexpression also decreases the rate of CE synthesis without affecting the acyl-CoA:cholesterol acyltransferase and neutral cholesterol ester hydrolase activities measured in vitro. In addition, it does not affect the transport of LDLderived cholesterol to the PM [113]. Because the SCP-2 overexpression stimulates cholesterol transport from ER to PM and stimulates secretion of cholesterol with HDL in hepatoma cells, the SCP-2 overexpression in L-cell fibroblasts inhibits cholesterol efflux to HDL at HDL concentration below $100 \mu \mathrm{g} / \mathrm{ml}$ [63]. Possible clue to explanation of negative effect of SCP-2 on cholesterol efflux is the inverse relationship between the expression of SCP-2 and liver fatty acid-binding protein (L-FABP aka FABP1). L-FABP is a hepatic cytosolic protein that binds long-chain fatty acids and other hydrophobic molecules including cholesterol. L-FABP protein level is twice higher in hepatocytes from SCP-2/SCP$\mathrm{x}$ knockout compared to hepatocytes from normal mouse. Cholesterol efflux to HDL in hepatocytes from SCP-2/SCP$\mathrm{x}$ knockout mouse is $35 \%$ higher than in normal mouse hepatocytes. However, cholesterol efflux in hepatocytes from $\mathrm{L}-\mathrm{FABP}^{-/-} / \mathrm{SCP}-2 / \mathrm{SCP}-\mathrm{x}^{-/-}$mouse is decreased compared to hepatocytes from WT mouse that indicates positive effect of L-FABP on the cholesterol efflux [114]. Thus, overexpression of SCP-2 might possibly repress the expression of L-FABP and inhibit the efflux. Besides cytoplasmic localization, SCP-2 and L-FABP are found on the PM in close proximity to SRBI. Some data suggest that L-FABP promotes uptake of HDL cholesterol by SR-BI in mouse hepatocytes [115].

3.4. Caveolae Cholesterol Is Actively Consumed in Cholesterol Efflux from the Cells. Caveolae are cholesterol-rich microdomains of PM formed by caveolin protein that are 
likely assembled in Golgi and transported to the PM. Both the newly synthesized and derived from LDL cholesterol pools first appear in caveolae and then they spread to non-caveolae areas of PM in human fibroblasts [116, 117]. In fibroblasts, at least $70 \%$ of cholesterol from LDL is quickly transported to caveolae domains of PM by a Golgi-dependent pathway. This path does not depend on the functional cytoskeleton. The rest of cholesterol from LDL is transported from lysosomes to the ER. This path is not Golgi-dependent and is inhibited by the disruption of actin filaments that suggests vesicular transport [4]. In yeast, contrary, newly synthesized ergosterol, the major yeast sterol, first appears mostly in non-raft fraction of PM. Then it equilibrates with raft sterol in about $1 \mathrm{~h}$.

The caveolar cholesterol is the primary PM cholesterol that is effluxed by the fibroblasts to plasma or HDL [116]. The nascent HDL produced by cholesterol efflux to apoAI by human embryonic kidney (HEK) 293 cells that express ABCA1 or mouse BMDMs are closer in cholesterol:PC and SM:PC ratio to rafts than to the PM fraction [118]. Caveolae formations start with expression of integral membrane proteins caveolin-1 or caveolin -2 in ER followed by their oligomerization (7-14 molecules of caveolin) and COPII-dependent transportation to the Golgi. The oligomer size in the Golgi increases to 18-25 molecules of caveolin and the oligomer-associated membrane saturates with cholesterol molecules. Then the cholesterol-rich complex is transported from trans-Golgi network to PM by four phosphate-adapter protein (FAPP1, FAPP2) secretory vesicles. Phosphatidylinositol-4-phosphate (PI4P), the prevalent phosphoinositide species in Golgi membrane, and small GTPases ARF1 play the essential role in the formation of these secretory vesicles [119]. Caveolin family consists of three genes. They share the property of insertion into the lipid membrane, the generation of oligomers with the formation of specific lipid rafts caveolae, which are cholesterol-enriched flask-shaped membrane invaginations of 50-100 nm in size [119]. Caveolins bind cholesterol and make specific pool of cholesterol on the PM [120, 121]. Caveolin-1 interacts with SCP-2 protein in caveolae in PM as well in cytosolic caveolar vesicles and caveolin/chaperone complexes of L-cells fibroblasts [121]. Cholesterol efflux to plasma is increased in fibroblasts transfected by caveolin-1 [38]; however the effect of the caveolin-1 overexpression on the efflux is not observed in CHOP cells [36]. Caveolins are not expressed in RAW 264.7 cells and caveolin-1 expression stimulates cholesterol efflux to HDL but not to apoA-I or to plasma $[17,36,122]$. In THP-1-derived macrophages, the knockdown of caveolin1 by antisense DNA inhibits cholesterol efflux to apoA-I with minimal effect on the efflux of PL [37]. A reduced cholesterol efflux to serum is observed in Cos-1 cells transfected with dominant-negative mutant of caveolin-1 that is not transported to the PM [123]. Transfection to express caveolin-1 in HepG2, hepatocyte cell line that does not express caveolin-1, results in an increased efflux to human plasma or apoA-I but not to cyclodextrin [36].

Contrary to stimulatory effect of caveolin-1 on cholesterol efflux observed in the most other cell cultures, mouse embryonic fibroblasts from caveolin-1 knockout mouse have an increased efflux to apoA-I compared to the cells from wild type animal. Induction of ABCA1 gene expression by LXR agonist increases this difference. Caveolin-1 disruption partly prevents apoA-I from its internalization into the cells and degradation. It is proposed that high curvature of the PM at the neck of caveolae is an attractive site for apoA-I binding to PM and the bound apoA-I is subjected to uptake and degradation [124].

\section{Molecular Mechanisms of Cholesterol Efflux}

Four major pathways mediate cholesterol efflux from cell plasma membrane and the contribution of the particular pathway varies depending on the cell type and extracellular acceptor nature (Table 2). These pathways include two ABCtransporters, ABCA1 and ABCG1, along with SR-BI and passive aqueous diffusion [125]. All the proteins mediating efflux from PM to extracellular acceptor are also involved in the intracellular cholesterol traffic and cholesterol distribution between various intracellular pools. The aqueous diffusion occurs for any cells; however, its contribution to the total cholesterol efflux for most of the type of cells is relatively small. All three proteins interact with other proteins at cholesterol efflux and the most significant pairs are given on Figure 2.

4.1. Aqueous Diffusion. Cholesterol can diffuse between membranes of cells, liposomes, and emulsions through the aqueous phase. The efficiency of nondirectional diffusional transfer of cholesterol is determined by the cholesterol capacity of the membrane and the kinetic factors-the rate of desorption and the concentration gradient. Cholesterol molecules desorbed from membranes are absorbed by various acceptors: plasma lipoproteins, plasma albumin and globulins, liposomes, and microemulsions, as well as specific molecules such as cyclodextrins [126].

When measuring cholesterol exchange rate between donor $(6.25 \mathrm{mg} / \mathrm{ml})$ and acceptor $(0.4-9.0 \mathrm{mg} / \mathrm{ml})$ single layer liposomes consisting of $20 \%$ cholesterol and $80 \%$ phospholipids that were separated by a dialysis membrane, the kinetics corresponded to a first-order reaction, which is characteristic of cholesterol transfer by the mechanism of free diffusion ((1) and (2)). The rate-limiting step in the exchange was the rate of cholesterol desorption [127]. However, this behavior is characteristic only for low concentrations of vesicles $(<3 \mathrm{mM})$. The kinetics of the second order, characteristic for the collision mechanism, is observed at high cholesterol concentrations [128] ((3)-(4)):

$$
\begin{aligned}
\mathrm{D}-\mathrm{Ch} & \longleftrightarrow \mathrm{D}+\mathrm{Ch}, \\
\mathrm{A}+\mathrm{Ch} & \longleftrightarrow \mathrm{A}-\mathrm{Ch}, \\
\mathrm{D}-\mathrm{Ch}+\mathrm{A} & \longleftrightarrow \mathrm{D}-\mathrm{Ch}-\mathrm{A}, \\
\mathrm{D}-\mathrm{Ch}-\mathrm{A} & \longleftrightarrow \mathrm{D}+\mathrm{A}-\mathrm{Ch},
\end{aligned}
$$

where $\mathrm{A}$ is the acceptor, $\mathrm{Ch}$ is the cholesterol, and D is the donor. 


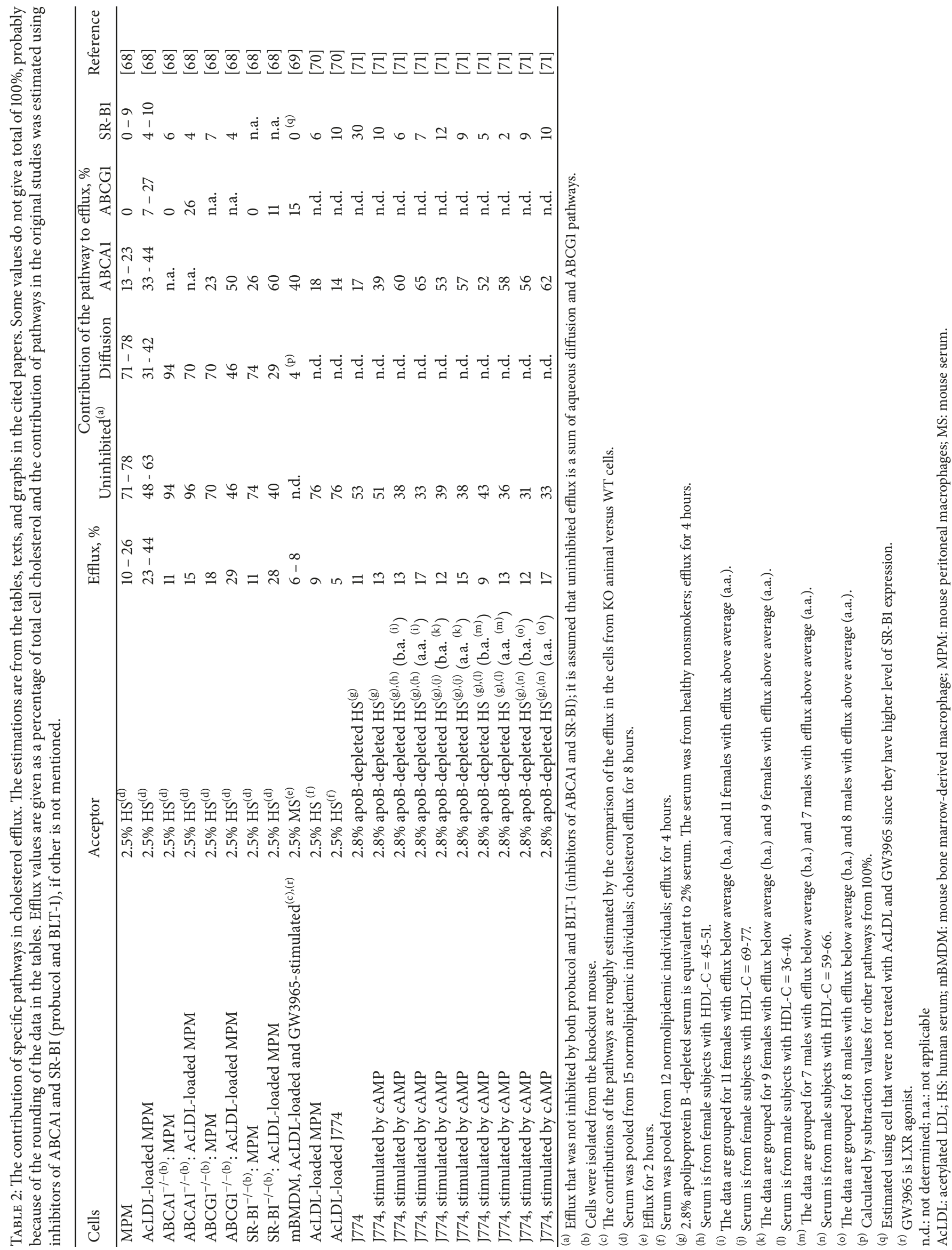




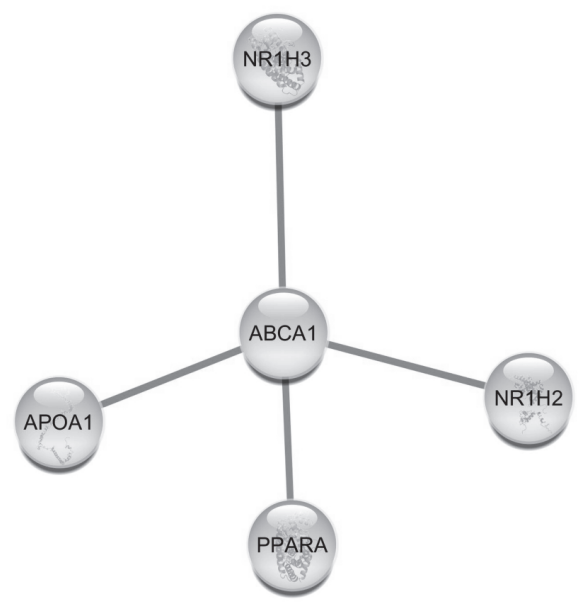

(a)

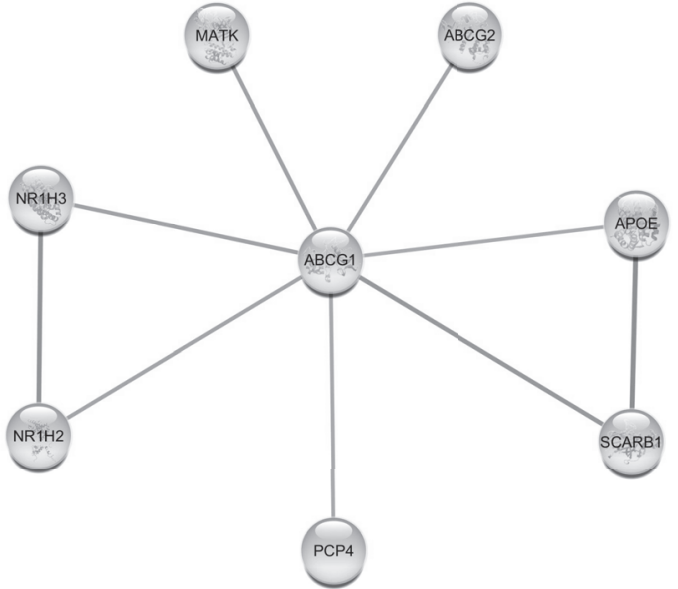

(b)

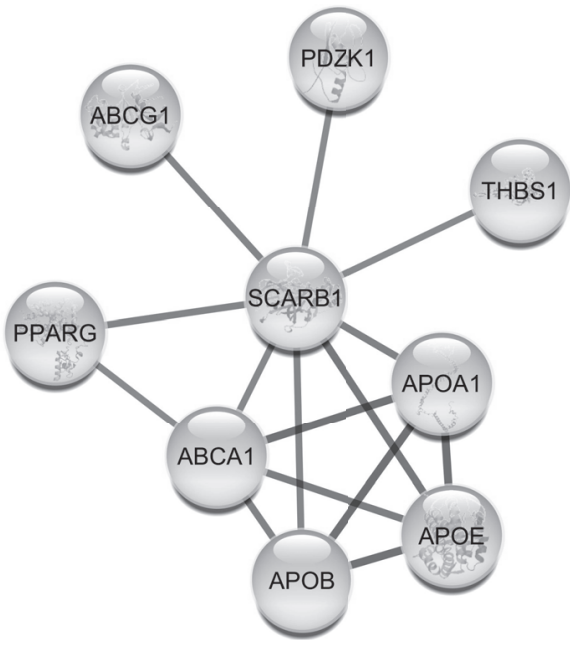

(c)

FIGURE 2: Protein-protein interactions involved in cholesterol efflux by cholesterol transporters. Data for human ABCA1 (a), ABCG1 (b), and SR-B1 (SCARB1) (c) molecules were imported from STRING database with Cytoscape STRING plugin. Confidence cutoff for interactions was chosen as $0.95,0.7$, and 0.8 , respectively. (a) apoA-I is the major protein component of HDL; PPARA is a nuclear receptor, transcription factor, key regulator of lipid metabolism; NR1H2 is an oxysterols receptor LXR-beta-nuclear receptor that regulates cholesterol uptake; $\mathrm{NR} 1 \mathrm{H} 3$ is an oxysterols receptor LXR-alpha-nuclear receptor that regulates homeostasis and cholesterol uptake. (b) NR1H3 and NR1H2 are mentioned above; MATK is a megakaryocyte-associated tyrosine-protein kinase that could play a significant role in the signal transduction of hematopoietic cells. PCP4 is a Purkinje cell protein 4 that plays an important role in synaptic plasticity, regulating calmodulin function; ABCG2 is an urate exporter that is able to mediate the export of protoporhyrin IX and implicated in the efflux of numerous drugs and xenobiotics; apoE is an apolipoprotein that mediates the binding, internalization, and catabolism of lipoprotein particles; SR-B1 (SCARB1) is a receptor for different ligands, receptor for HDL. (c) apoA-I and apoE are mentioned above; ABCA1 and ABCG1 play a role in HDL metabolism; apoB is a major protein component of chylomicrons, VLDL, and LDL; PPARG is a nuclear receptor that controls the peroxisomal beta-oxidation pathway of fatty acids and regulates adipocyte differentiation and glucose homeostasis; PDZK1 is a PDZ domain containing scaffolding protein; THBS1 is a thrombospondin-1, adhesive glycoprotein, that mediates cell-to-cell and cell-to-matrix interactions, binds heparin.

When measuring the rate of intermembrane cholesterol transfer, the following deviations from the diffusion mechanism were observed: (1) the rate of cholesterol transfer from erythrocytes to acceptors was inversely related to the size of the acceptor; (2) the rate of cholesterol transfer from erythrocytes to the erythrocyte ghosts increased with the addition of plasma, while the opposite effect could be expected due to competition between ghosts and plasma components that act as cholesterol acceptors; (3) the rate of transfer decreased upon the dilution of the mixture of erythrocytes and ghosts but did not obey the second-order kinetics; (4) cholesterol in the membranes of the bovine retina rod cells is not in equilibrium with the cholesterol of the plasma and its content increased after incubation with plasma; (5) the deviation of transfer kinetics from the diffusion model can not be explained by the presence of a 
non-stirred layer, since the transfer rate of lysolecithin was three orders of magnitude higher. The authors suggested a combined model of the primary activation of cholesterol $\left(\mathrm{Ch}^{\prime}\right)$ in the donor membrane in the first-order kinetics followed by the formation of the donor-acceptor complex in second-order kinetics [129] ((5)-(7)):

$$
\begin{aligned}
\mathrm{D}-\mathrm{Ch} & \longleftrightarrow \mathrm{D}-\mathrm{Ch}^{\prime}, \\
\mathrm{D}-\mathrm{Ch}^{\prime}+\mathrm{A} & \longleftrightarrow \mathrm{D}-\mathrm{Ch}^{\prime}-\mathrm{A}, \\
\mathrm{D}-\mathrm{Ch}^{\prime}-\mathrm{A} & \longleftrightarrow \mathrm{D}+\mathrm{Ch}^{\prime}-\mathrm{A} .
\end{aligned}
$$

A similar model was proposed for the transfer of cholesterol from erythrocytes to $\mathrm{HDL}_{2}, \mathrm{HDL}_{3}$, or LDL, when the cholesterol exchange rate is nonlinearly dependent on the concentration of lipoproteins at their low concentrations and reaches a plateau at high concentrations of lipoproteins. In this model, the cholesterol transfer from erythrocytes is determined by the collision mechanism at low concentrations of acceptors, while the diffusion of cholesterol in the erythrocyte membrane to the specific sites of lipoprotein adsorption becomes rate limiting at high concentrations of acceptors [130].

However, the transfer of cholesterol between vesicles and reconstituted HDL (rHDL) was not consistent with aqueous diffusion or collision mechanisms. It was suggested that apoA-I of rHDL interacts with vesicles, which facilitates the transfer of cholesterol, and the interaction depends on the conformation of the apolipoprotein. This model was consistent with the data on the transfer of cholesterol from vesicles to rHDL with different amounts of apoA-I and explained the effect of the composition of rHDL and vesicles on the rate of cholesterol transfer [131]. Opposite, the collision mechanism at cholesterol exchange between vesicles and rHDL (apoA-I-containing nanodiscs) was discarded in the work of Matsuzaki et al. The authors postulated the importance of the diffusion mechanism with cholesterol dissociation from the vesicles as a rate-limiting step of the cholesterol transfer. Interestingly, the rate of dissociation of cholesterol from rHDL bilayer was higher than the rate of dissociation from the bilayer of liposomes with similar composition [132]. The authors suggested that it might be explained by denser bilayer packing in rHDL. Another reason may be the appearance of "active" cholesterol in the nanodiscs due to the heterogeneity of the distribution of cholesterol in discoidal lipoproteins. Such heterogeneity we found in the reconstituted particles containing various apolipoproteins [133].

4.2. ABCA1. ABCA1 is localized in the plasma membrane and in late and early endosomes [134]. The LE-located ABCA1 seems to assist in efflux of LE pool of cholesterol [135]. It was reported that overexpression of $\mathrm{ABCAl}$ prevents the accumulation of cholesterol in LE and lysosomes in NPC1deficient, but not NPC2-deficient cells [136]. Another study demonstrated that ABCA1 participates in the cholesterol traffic from PM to ER. The reduction of the ability of the membrane to retain cholesterol after treatment by sphingomyelinase leads to rapid flow of PM cholesterol inside the cell, its esterification, and inhibition of cholesterol synthesis [72]. This PM to ER flow is mediated by ABCA1 by about $50 \%$ in mouse embryonic fibroblasts. ABCA1 mutant lacking ATPase activity is unable to provide the PM to ER cholesterol transport [137]. Bioinformatics analysis of functional and physical interactions of ABCA1 reveals apoA-I and several nuclear receptors (acting as transcriptional factors) as major partners/modulators of ABCA1 (Figure 2(a)).

ABCA1 structure includes two hydrophobic transmembrane domains, each containing six $\alpha$-helices, and two hydrophilic domains, called nucleotide-binding folds [138]. ATP hydrolysis in two hydrophilic domains of ABCA1 results in a change in the protein conformation accompanied by the transfer of the transported molecule to the outer part of the membrane. ABCA1 transports phosphatidylcholine, phosphatidylinositol $(4,5)$ bis-phosphate (PIP2) [44], and less efficiently phosphatidylserine and sphingomyelin [139]. Recently we described the existence of two types of putative cholesterol-binding motifs in ABCA1 and their involvement into binding of cholesterol molecules differently immersed in lipid bilayer (Dergunov et al., 2018 submitted).

An increase in the cholesterol content in the cell, firstly, inhibits ubiquitination and subsequent degradation of $A B C A 1$, thereby increasing the level of the ABCA1 protein $[140]$ and, secondly, induces the expression of genes involved in cholesterol efflux [68]. ABCA1 molecules undergo palmitoylation of cysteine residues $3,23,1110$, and 1111 . In the absence of these modifications, the transporter molecules remain inside the cell. The absence of any of these palmitoylation reduces cholesterol efflux from the cells [141]. Cholesterol efflux mediated by $\mathrm{ABCA1}$ results in the formation of discoidal HDL, containing two, three, or four molecules of apoA-I per particle. Nascent HDLs are heterogeneous in size and composition and contain the main classes of lipids present in plasma membrane. In addition to discoidal HDL, ABCAl-mediated efflux generates lipid-poor apoA-I with one molecule of apolipoprotein [142, 143]. Cholesterol of the plasma membrane can be replenished from endosomal compartments in the minute range [144]. Importantly, a direct relation between ABCA1-mediated cellular cholesterol efflux and arterial-wall thickness exists that suggests the inhibition of atherosclerosis progression by efflux increase before the manifestation of symptomatic cardiovascular disease [145]. However, some controversy exists on the contribution of both common and rare ABCA1 variants and levels of HDL cholesterol to risk of ischemic heart disease in the general population [146, 147]. Interestingly, stimulation of macrophage mitochondrial ATP production resulted in the increase of ABCAl expression and cholesterol efflux with a concomitant decrease in aortic sinus lesion area in atherosclerosis-prone mice, despite no changes in HDL cholesterol [148]. Besides, the protective effect of ABCA1 pathway activation in reactive astrocytes at ischemic stroke has been suggested [149].

The nature of the molecular interaction between various cholesterol acceptors and ABCA1 is controversial, and two alternative models suggesting a direct protein-protein interaction or indirect association have been proposed. According to the first model, the apoA-I and ABCA1 molecules interact 
$[150,151]$. In the second two-site model, besides the direct ABCA1-apoA-I interaction, the existence of much more pronounced association of the apolipoprotein with the lipid phase near the transporter molecule is suggested [152]. Both models recognize the significant contribution of the interaction of apoA-I and membrane lipids in the ABCA1-mediated cholesterol efflux. ABCA1 translocates phospholipids to the exofacial leaflet of the plasma membrane bilayer that creates membrane tension. Apolipoprotein-lipid interaction results in the decrease of this tension [139]. The introduction of the amphipathic helix of apoA-I into protrusion on the plasma membrane [153], similar to the solubilization by apolipoprotein of multilayer vesicles [143, 154], leads to the dissociation of cholesterol and phospholipids in apolipoproteinlipid complexes from the plasma membrane. Note that the complexes primarily include cholesterol, not contained in the rafts [155].

According to the model of the direct interaction between the apolipoprotein and the transporter, the mechanism of the ABCA1-mediated efflux of cholesterol includes the following steps: (1) diffusion of the transporter in a membrane and ATP-dependent lipid flopping; (2) dimerization of the transporter and fixation of the dimer in the membrane involving the cytoskeleton; (3) the interaction of lipid-free apoA-I with the dimer, followed by apolipoprotein-lipid interaction, dissociation of the apolipoprotein-transporter complex, and the release of the lipid-laden apoA-I into the extracellular environment; (4) dissociation of the dimer with closing of the ABCA1 cycle [156]. The alternative model questions the exclusiveness of the direct interaction of apoA-I and ABCA1. Experiments on human fibroblasts have shown that most (90\%) of the apoA-I molecules bind to the HCBS (high capacity binding site), and not to ABCA1. Interestingly, HCBS is not a part of the lipid rafts. HCBS mainly consists of phosphatidylcholine, and not sphingomyelin, and is not associated with caveolin-1. PIP2 plays significant role in the binding of apoA-I to the membrane, and PIP2 is transferred to the exofacial leaflet of plasma membrane by the floppase activity of ABCA1 [44]. The disruption of the interaction of the apolipoprotein with HCBS significantly reduced cholesterol efflux on apoA-I. According to this model, ABCA1 generates HCBS, and the direct interaction of apoA-I with ABCA1 could stabilize the structure of ABCA1 [152, 157]. Wang et al. suggested the existence in ABCA1 structure a nonspecific low-affinity site of binding with apoA-I. This site serves as a chaperone in the unfolding of the $\mathrm{N}$-terminus of the apolipoprotein, which is an important process in the formation of HDL [158].

4.3. ABCG1. ABCG1 is another, along with ABCA1, ATPbinding cassette transporter that participates in cholesterol efflux in macrophages (Table 2). There is some inconsistency in the data on ABCG1 localization. In a recent study in $\mathrm{CHO}$ and HeLa cell lines that were transfected to stably express ABCG1 fused with Myc tag or green fluorescent protein (GFP), the transporter was distributed between PM and ER pools. The PM pool increased after loading the cells with cholesterol [159]. In another study of ABCG1-GFP fusion, expressed in HeLa cells, the transporter was observed in PM and late endosomes [160]. However, another group, using several approaches, found that intracellular endosomes are the only sites of ABCG1 localization, and ABCG1 is not observed in PM neither in murine peritoneal macrophages, nor in CHO, Cos-7, and HEK293 cells transfected with untagged or FLAG-tagged ABCG1 [161, 162].

Structurally, ABCG1 contains a hydrophobic transmembrane domain of six $\alpha$-helices and a hydrophilic nucleotidebinding folds domain. It resembles half of $\mathrm{ABCA1}$ transporter and is called sometimes the half-transporter [163]. ABCG1 is palmitoylated at positions $26,150,311,390$, and 402 . The palmitoylation at Cys311 is critical for the localization of ABCG1 at the plasma membrane and for ABCG1-dependent cholesterol efflux, while palmitoylation at other positions is not essential for these processes [164]. In addition, tyrosine Y667 of cholesterol-binding motif of ABCG1 is functionally important: the substitution of this tyrosine reduced the efflux [165]. Interestingly, ABCG1, in addition to cholesterol, transports sphingomyelin, and different structural motifs are responsible for binding of these lipids. It was suggested that sphingomyelin stimulates cholesterol efflux by ABCG1 via activation of its ATPase activity [166]. The cholesterolbinding motif, which is important for the cholesterol efflux activity of ABCG1, was also found in half-transporters ABCG4 and ABCG8 that mediate cholesterol efflux [165]. We predicted the presence of two types of cholesterolbinding motifs in the ABCG1 structure that mediate the transport of different pools of cholesterol (Dergunov et al., 2018 submitted).

ABCG1 expression results in the increase of the rate of cholesterol desorption and in the increase of cholesterol pool available for both efflux and esterification [167, 168]. The major partners/modulators of ABCG1 that were determined by bioinformatics analysis are presented on Figure 2(b). The expression of ABCG1 is associated with an increase in the efflux of cholesterol on $\mathrm{HDL}_{2}, \mathrm{HDL}_{3}, \mathrm{LDL}, \mathrm{SUV}$, and rHDL, but not on lipid-free apoA-I and does not affect cholesterol influx $[167,169]$. Despite the massive lipid accumulation in hepatocytes and in macrophages in mice with targeted disruption of $A b c g 1$ gene and the promotion of cholesterol efflux to HDL in human ABCG1-transgenic mice, the plasma lipid level did not change in both cases [169]. Moreover, ABCG1 in the arterial wall might possess a proatherogenic effect independently of any modulation of HDL-C level [170]. ABCG1 is not expected to attenuate foam cell formation in early atherosclerosis lesions in humans [171] but could protect from atherosclerosis by preserving vascular endothelium from dietary cholesterol-induced dysfunction [172]. There is no specific binding of the transporter to the lipoprotein in ABCG1-mediated efflux of cholesterol. For SUV, LDL, and rHDL discs as cholesterol acceptors, the kinetics of the ABCG1-mediated efflux corresponds to the aqueous diffusion mechanism, and ABCG1 increases the pool of active cholesterol available for efflux. The kinetics of efflux with $\mathrm{HDL}_{2}$ and $\mathrm{HDL}_{3}$ deviates from the kinetics for diffusion, presumably due to the shielding by apolipoproteins of cholesterol-accepting phospholipid patches. In experiments with BHK cells, the size of the pool of cholesterol available for efflux on $\mathrm{HDL}_{3}$ as an acceptor was about $20 \%$ of total 
cell cholesterol. The expression of ABCG1 increased the pool size by $6-12 \%$ [167]. Neufeld et al. also observed the increase of the pool of active cholesterol available for efflux. Both PM- and late endosome-localized ABCG1 contributed to the stimulation of cholesterol efflux. LE-localized ABCG1 stimulated cholesterol transfer from the LE to PM, perhaps by direct interaction between LE and PM membranes, and/or in a process, mediated by nonvesicular cytosolic cholesterol carrier proteins such as OSBP [160].

4.4. SR-B1. Scavenger receptor class B type 1 (SR-B1) belongs to the superfamily of CD36 scavenger receptors. SR-B1 is highly expressed in the liver, where it participates in RCT [173], in steroidogenic tissues (adrenal gland and ovary) and mammary gland during lactation [174]. According to the bioinformatics analysis of physical and functional interactions, the most important SR-B1 protein partners are apolipoproteins and proteins involved in HDL metabolism, nuclear receptor PPAR $\gamma$, a scaffold protein PDZ domain containing 1 (PDZK1) that controls SR-B1 level in liver [175, 176], and thrombospondin-1 (THBS1), a subunit of disulfidelinked homotrimeric protein that binds components of extracellular matrix and mediates cell-to-cell interactions (Figure 2(c)). The expression of THBS1 is downregulated by HMG-CoA reductase inhibitor lovastatin [177].

SR-B1 molecule contains two hydrophobic domains and an extracellular glycosylated domain [178]. The cytoplasmic C-terminal domain of SR-B1 binds to PDZ domains of PDZK1 protein [179]. SR-B1, being an HDL receptor, selectively transfers cholesteryl ester molecules contained in HDL into a cell without endocytosis and degradation of HDL particle [180]. In addition to HDL, the receptor exhibits broad ligand specificity for LDL, ox-LDL, and VLDL [181]. As the diameter of HDL increases, the maximum binding value $B_{\max }$ increases, and the value of the dissociation constant $K_{\mathrm{d}}$ decreases; that is, the large HDL are the best ligands for SRB1 [182]. Nevertheless, HDL binding to SR-B1 is about 150fold lower than the binding of LDL to LDL receptor. The weaker binding and the faster dissociation of HDL from SRB1 can explain why HDL does not undergo endocytosis. The isotherm of binding of HDL to SR-B1 on the cell surface most corresponds to the presence in SR-B1 of one high affinity and one low-affinity binding sites. The rate of cholesterol transfer from HDL increases proportionally with their $\mathrm{CE}$ content. The current model of SR-B1 action suggests CE transfer between HDL and PM through the hydrophobic channel along the concentration gradient [183]. This mechanism can be viewed as a facilitated diffusion. It is assumed that the transfer of cholesterol similarly occurs through the hydrophobic channel. However, SR-B1 also redistributes the membrane pools of cholesterol, as evidenced by the increase in the amount of cholesterol available for oxidation with cholesterol oxidase [184]. Perhaps the cholesterol-binding motifs predicted by us in the structure of SR-B1 (Dergunov et al., 2018 submitted) are responsible for the redistribution of cholesterol in the membrane.

It is important that the influx of the CE correlates with the binding of HDL, while the efflux of cholesterol continues to increase after the receptor is saturated with the lipoprotein.
Perhaps this is due to the SR-B1-induced redistribution of the cholesterol membrane pools in favor of the efflux by the diffusion mechanism or because of collisions [182]. Some artificial chimeric receptors with partial substitution of the SR-B1 sequence by the sequence of another SR-B class receptor CD36 retain the ability to efflux cholesterol even with significantly decreased binding of HDL. These chimeric receptors also retained the ability of SR-B1 to increase the cholesterol pool accessible to cholesterol oxidase [185]. Functional analysis of SR-B1 mutants shows that various activities of SR-B1, such as CE influx, cholesterol efflux, redistribution of membrane pools of cholesterol, and HDL binding, are partly independent due to the presence of mutations that affect some of the activities and do not affect others [185-188].

\section{Conclusion}

The gradient of cholesterol concentration between plasma membrane and the membranes of intracellular organelles is maintained by constant redistribution of the cholesterol in energy-dependent vesicular traffic and energy-independent transport by lipid transfer proteins. Vesicular traffic plays just a minor role in the cholesterol transfer from the site of synthesis (ER) to PM; however it plays the central role in endocytosis of LDL-delivered cholesterol. The fractions of fast- and slow-exchanging pools of cholesterol are determined by the membrane lipid composition and the kinetics of the association and dissociation of the cholesterol complexes with other membrane lipids, such as in lipid rafts, which are stable in some range of the cholesterol/phospholipid ratio in the membrane. Just partly exposed into the aqueous phase cholesterol molecules that are not incorporated to lipid complexes are available to the fast escape from the membrane, which is the rate-limiting event in the aqueous diffusion pathway in cholesterol efflux. The facilitated diffusion along the concentration gradient through the hydrophobic tunnel occurs in the cholesterol efflux mediated by SR-B1. ABCG1mediated cholesterol efflux is supposedly determined by the increase of the pool of active cholesterol that is available for the transfer from the membrane to lipoproteins by aqueous diffusion. Active cholesterol may originate from the specific cholesterol-binding motifs in ABCG1 structure at membrane interface. ABCA1-mediated efflux occurs due to the floppase activity of the transporter powered by the hydrolysis of ATP. ApoA-I binding to the cell surface is essential in the ABCAl-mediated cholesterol efflux; however it is not clear yet whether apoA-I binds to ABCA1 or to the lipid phase of plasma membrane. A number of intracellular processes determine the availability of the cholesterol for the efflux to extracellular acceptors.

\section{Abbreviations and Designations}
ACAT: Acyl-coenzyme A:cholesterol acyltransferase
acLDL: Acetylated low-density lipoprotein
apoA-I: Apolipoprotein A-I
CE: Cholesteryl ester
ER: Endoplasmic reticulum 


$\begin{array}{ll}\text { ERC: } & \text { Endocytic recycling compartment } \\ \text { HCBS: } & \text { High capacity binding site } \\ \text { HDL: } & \text { High density lipoprotein } \\ \text { HEK: } & \text { Human embryonic kidney } \\ \text { HMGCR-3: } & \text { Hydroxy-3-methylglutaryl coenzyme A } \\ & \text { reductase } \\ \text { LD: } & \text { Lipid droplet } \\ \text { LDL: } & \text { Low-density lipoprotein } \\ \text { LDLR: } & \text { LDL receptor } \\ \text { LE: } & \text { Late endosome } \\ \text { L-FABP: } & \text { Liver fatty acid-binding protein } \\ \text { LTP: } & \text { Lipid transfer protein } \\ \text { LXR: } & \text { Liver X receptor } \\ \text { MCSs: } & \text { Membrane contact sites } \\ \text { OSBP: } & \text { Oxysterol-binding protein } \\ \text { PI4P: } & \text { Phosphatidylinositol-4-phosphate } \\ \text { PIP2: } & \text { Phosphatidylinositol (4,5) bis-phosphate } \\ \text { PL: } & \text { Phospholipid } \\ \text { PM: } & \text { Plasma membrane } \\ \text { rHDL: } & \text { Reconstituted high density lipoproteins } \\ \text { SCAP: } & \text { SREBP cleavage-activating protein } \\ \text { SCP-2: } & \text { Sterol carrier protein 2 } \\ \text { SM: } & \text { Sphingomyelin } \\ \text { SR-B1: } & \text { Scavenger receptor class B type 1 } \\ \text { SREBP: } & \text { Sterol regulatory element-binding protein } \\ \text { STARD: } & \text { StAR related lipid transfer domain } \\ & \text { containing proteins } \\ \text { TGN: } & \text { Trans-Golgi network } \\ \text { VAP: } & \text { Vesicle-associated membrane } \\ & \text { protein-associated protein } \\ \text { VLDL: } & \text { Very density lipoprotein. } \\ & \\ \text { Hel } & \\ & \end{array}$

\section{Disclosure}

The article does not contain any studies with human or animal subjects performed by any of the authors.

\section{Conflicts of Interest}

Dmitry Y. Litvinov, Eugeny V. Savushkin, and Alexander D. Dergunov declare that they have no conflicts of interest.

\section{Acknowledgments}

This study was supported by the grant from the Russian Fund for Basic Research (Grant 16-04-00067).

\section{References}

[1] M. Ouimet, E. J. Hennessy, C. Van Solingen et al., "MiRNA targeting of oxysterol-binding protein-like 6 regulates cholesterol trafficking and efflux," Arteriosclerosis, Thrombosis, and Vascular Biology, vol. 36, no. 5, pp. 942-951, 2016.

[2] A. D. Dergunov, E. A. Garaeva, E. V. Savushkin, and D. Y. Litvinov, "Significance of Lipid-Free and Lipid-Associated ApoA-I in Cellular Cho-lesterol Efflux," Current protein \& peptide science, vol. 18, no. 1, pp. 92-99, 2017.

[3] D. Y. Litvinov, E. V. Savushkin, E. A. Garaeva, and A. D. Dergunov, "Cholesterol efflux and reverse cholesterol transport:
Experimental approaches," Current Medicinal Chemistry, vol. 23, no. 34, pp. 3883-3908, 2016.

[4] L. Liscum and N. J. Munn, "Intracellular cholesterol transport," Biochimica et Biophysica Acta (BBA) - Molecular and Cell Biology of Lipids, vol. 1438, no. 1, pp. 19-37, 1999.

[5] D. E. Warnock, C. Roberts, M. S. Lutz, W. A. Blackburn, W. W. Young Jr., and J. U. Baenziger, "Determination of plasma membrane lipid mass and composition in cultured Chinese hamster ovary cells using high gradient magnetic affinity chromatography," The Journal of Biological Chemistry, vol. 268, no. 14, pp. 10145-10153, 1993.

[6] Y. Lange, M. H. Swaisgood, B. V. Ramos, and T. L. Steck, "Plasma membranes contain half the phospholipid and $90 \%$ of the cholesterol and sphingomyelin in cultured human fibroblasts," The Journal of Biological Chemistry, vol. 264, no. 7, pp. 37863793, 1989.

[7] Y. Lange, F. Strebel, and T. L. Steck, "Role of the plasma membrane in cholesterol esterification in rat hepatoma cells," The Journal of Biological Chemistry, vol. 268, no. 19, pp. 13838-13843, 1993.

[8] Y. Lange, J. Ye, M. Rigney, and T. L. Steck, "Regulation of endoplasmic reticulum cholesterol by plasma membrane cholesterol," Journal of Lipid Research, vol. 40, no. 12, pp. 2264-2270, 1999.

[9] I. Levental and S. L. Veatch, "The Continuing Mystery of Lipid Rafts," Journal of Molecular Biology, vol. 428, no. 24, pp. 47494764, 2016.

[10] A. Y. Andreyev, E. Fahy, Z. Guan et al., "Subcellular organelle lipidomics in TLR-4-activated macrophages," Journal of Lipid Research, vol. 51, no. 9, pp. 2785-2797, 2010.

[11] B. W. Wattenberg and D. F. Silbert, "Sterol partitioning among intracellular membranes. Testing a model for cellular sterol distribution," The Journal of Biological Chemistry, vol. 258, no. 4, pp. 2284-2289, 1983.

[12] A. Sokolov and A. Radhakrishnan, "Accessibility of cholesterol in endoplasmic reticulum membranes and activation of SREBP2 switch abruptly at a common cholesterol threshold," The Journal of Biological Chemistry, vol. 285, no. 38, pp. 29480-29490, 2010.

[13] A. Radhakrishnan, J. L. Goldstein, J. G. McDonald, and M. S. Brown, "Switch-like Control of SREBP-2 Transport Triggered by Small Changes in ER Cholesterol: A Delicate Balance," Cell Metabolism, vol. 8, no. 6, pp. 512-521, 2008.

[14] M. P. Haynes, M. C. Phillips, and G. H. Rothblat, "Efflux of cholesterol from different cellular pools," Biochemistry, vol. 39, no. 15, pp. 4508-4517, 2000.

[15] N. Mukhamedova, A. Hoang, H. L. Cui et al., "Small GTPase ARF6 Regulates Endocytic Pathway Leading to Degradation of ATP-Binding Cassette Transporter A1," Arteriosclerosis, Thrombosis, and Vascular Biology, vol. 36, no. 12, pp. 2292-2303, 2016.

[16] W. Chen, Y. Sun, C. Welch et al., "Preferential ATP-binding cassette transporter Al-mediated cholesterol efflux from late endosomes/lysosomes," The Journal of Biological Chemistry, vol. 276, no. 47, pp. 43564-43569, 2001.

[17] N. Mukhamedova, G. Escher, W. D'Souza et al., "Enhancing apolipoprotein A-I-dependent cholesterol efflux elevates cholesterol export from macrophages in vivo," Journal of Lipid Research, vol. 49, no. 11, pp. 2312-2322, 2008.

[18] H. Ohvo-Rekilä, B. Ramstedt, P. Leppimäki, and J. Peter Slotte, "Cholesterol interactions with phospholipids in membranes," Progress in Lipid Research, vol. 41, no. 1, pp. 66-97, 2002. 
[19] Y. Lange and T. L. Steck, "Cholesterol homeostasis and the escape tendency (activity) of plasma membrane cholesterol," Progress in Lipid Research, vol. 47, no. 5, pp. 319-332, 2008.

[20] G. Drin, "Topological regulation of lipid balance in cells," Annual Review of Biochemistry, vol. 83, pp. 51-77, 2014.

[21] E. Sezgin, I. Levental, S. Mayor, and C. Eggeling, “The mystery of membrane organization: Composition, regulation and roles of lipid rafts," Nature Reviews Molecular Cell Biology, vol. 18, no. 6, pp. 361-374, 2017.

[22] D. Wüstner and K. Solanko, "How cholesterol interacts with proteins and lipids during its intracellular transport," Biochimica et Biophysica Acta (BBA) - Biomembranes, vol. 1848, no. 9, pp. 1908-1926, 2015.

[23] B. B. Johnson and A. P. Heuck, "Perfringolysin O structure and mechanism of pore formation as a paradigm for cholesteroldependent cytolysins," Subcellular Biochemistry, vol. 80, pp. 6381, 2014.

[24] Y. Lange, S. M. A. Tabei, J. Ye, and T. L. Steck, "Stability and stoichiometry of bilayer phospholipid-cholesterol complexes: Relationship to cellular sterol distribution and homeostasis," Biochemistry, vol. 52, no. 40, pp. 6950-6959, 2013.

[25] Y. Lange, J. Ye, and T. L. Steck, "Essentially all excess fibroblast cholesterol moves from plasma membranes to intracellular compartments," PLoS ONE, vol. 9, no. 8, 2014.

[26] A. Das, M. S. Brown, D. D. Anderson, J. L. Goldstein, and A. Radhakrishnan, "Three pools of plasma membrane cholesterol and their relation to cholesterol homeostasis," eLife, vol. 2014, no. 3, Article ID e02882, 2014.

[27] P. G. Yancey, W. V. Rodrigueza, E. P. C. Kilsdonk et al., "Cellular cholesterol efflux mediated by cyclodextrins: Demonstration of kinetic pools and mechanism of efflux," The Journal of Biological Chemistry, vol. 271, no. 27, pp. 16026-16034, 1996.

[28] M. Hao, S. X. Lin, O. J. Karylowski, D. W. Stner, T. E. McGraw, and F. R. Maxfield, "Vesicular and non-vesicular sterol transport in living cells: The endocytic recycling compartment is a major sterol storage organelle," The Journal of Biological Chemistry, vol. 277, no. 1, pp. 609-617, 2002.

[29] Y.-C. Lv, Y.-Y. Tang, J. Peng et al., "MicroRNA-19b promotes macrophage cholesterol accumulation and aortic atherosclerosis by targeting ATP-binding cassette transporter Al," Atherosclerosis, vol. 236, no. 1, pp. 215-e226, 2014.

[30] S. Bocchetta, P. Maillard, M. Yamamoto et al., "Up-regulation of the ATP-binding cassette transporter A1 inhibits hepatitis C virus infection," PLoS ONE, vol. 9, no. 3, Article ID e92140, 2014.

[31] H. Low, N. Mukhamedova, H. L. Cui et al., "Cytomegalovirus Restructures Lipid Rafts via a US28/CDC42-Mediated Pathway, Enhancing Cholesterol Efflux from Host Cells," Cell Reports, vol. 16, no. 1, pp. 186-200, 2016.

[32] F. Dong, Z. Mo, W. Eid, K. C. Courtney, and X. Zha, "Akt inhibition promotes ABCA1-mediated cholesterol efflux to apoA-I through suppressing mTORC1," PLoS ONE, vol. 9, no. 11, Article ID el13789, 2014.

[33] D. Wang, X. Yan, M. Xia et al., "Coenzyme Q10 promotes macrophage cholesterol efflux by regulation of the activator protein-1/miR-378/ATP-binding cassette transporter G1signaling pathway," Arteriosclerosis, Thrombosis, and Vascular Biology, vol. 34, no. 9, pp. 1860-1870, 2014.

[34] S. Lin, C. Zhou, E. Neufeld et al., "BIG1, a brefeldin A-inhibited guanine nucleotide-exchange protein modulates ATP-binding cassette transporter A-1 trafficking and function," Arteriosclerosis, Thrombosis, and Vascular Biology, vol. 33, no. 2, pp. e31-e38, 2013.
[35] L. Jennelle, R. Hunegnaw, L. Dubrovsky et al., "HIV-1 protein NEF inhibits activity of ATP-binding cassette transporter A1 by targeting endoplasmic reticulum chaperone calnexin," The Journal of Biological Chemistry, vol. 289, no. 42, pp. 2887028884, 2014.

[36] Y. Fu, A. Hoang, G. Escher, R. G. Parton, Z. Krozowski, and D. Sviridov, "Expression of Caveolin-1 Enhances Cholesterol Efflux in Hepatic Cells," The Journal of Biological Chemistry, vol. 279, no. 14, pp. 14140-14146, 2004.

[37] R. Arakawa, S. Abe-Dohmae, M. Asai, J.-I. Ito, and S. Yokoyama, "Involvement of caveolin-1 in cholesterol enrichment of high density lipoprotein during its assembly by apolipoprotein and THP-1 cells," Journal of Lipid Research, vol. 41, no. 12, pp. 19521962,2000

[38] C. J. Fielding, A. Bist, and P. E. Fielding, "Intracellular cholesterol transport in synchronized human skin fibroblasts," Biochemistry, vol. 38, no. 8, pp. 2506-2513, 1999.

[39] D. Gong, H.-P. Cheng, W. Xie et al., "Cystathionine $\gamma$ lyase(CSE)/hydrogen sulfide system is regulated by miR-216a and influences cholesterol efflux in macrophages via the PI3K/ AKT/ABCA1 pathway," Biochemical and Biophysical Research Communications, vol. 470, no. 1, pp. 107-116, 2016.

[40] B. Haidar, R. S. Kiss, L. Sarov-Blat et al., "Cathepsin D, a lysosomal protease, regulates ABCA1-mediated lipid efflux," The Journal of Biological Chemistry, vol. 281, no. 52, pp. 39971-39981, 2006.

[41] N. Ohoka, K. Okuhira, H. Cui et al., "HNF4 Increases LiverSpecific Human ATP-Binding Cassette Transporter A1 Expression and Cholesterol Efflux to Apolipoprotein A-I in Response to Cholesterol Depletion," Arteriosclerosis, Thrombosis, and Vascular Biology, vol. 32, no. 4, pp. 1005-1014, 2012.

[42] C. M. Ramírez, C. S. Lin, K. Abdelmohsen et al., "RNA binding protein HuR regulates the expression of ABCA1," Journal of Lipid Research, vol. 55, no. 6, pp. 1066-1076, 2014.

[43] A. Katsube, H. Hayashi, and H. Kusuhara, "Pim-1L Protects Cell Surface-Resident ABCA1 from Lysosomal Degradation in Hepatocytes and Thereby Regulates Plasma High-Density Lipoprotein Level," Arteriosclerosis, Thrombosis, and Vascular Biology, vol. 36, no. 12, pp. 2304-2314, 2016.

[44] K. Gulshan, G. Brubaker, H. Conger et al., "PI(4,5)P2 is Translocated by ABCA1 to the Cell Surface Where It Mediates Apolipoprotein A1 Binding and Nascent HDL Assembly," Circulation Research, vol. 119, no. 7, pp. 827-838, 2016.

[45] Q. Zhou, Y. Mei, T. Shoji et al., "Rho-associated coiled-coilcontaining kinase 2 deficiency in bone marrow-derived cells leads to increased cholesterol efflux and decreased atherosclerosis," Circulation, vol. 126, no. 18, pp. 2236-2247, 2012.

[46] M. D. Linder, M. I. Mäyränpää, J. Peränen et al., "Rab8 regulates ABCA1 cell surface expression and facilitates cholesterol efflux in primary human macrophages," Arteriosclerosis, Thrombosis, and Vascular Biology, vol. 29, no. 6, pp. 883-888, 2009.

[47] K. Nakaya, M. Ayaori, H. Uto-Kondo et al., "Overexpression of stearoyl-coenzyme A desaturase 1 in macrophages promotes reverse cholesterol transport," Biochimica et Biophysica Acta (BBA) - Molecular and Cell Biology of Lipids, vol. 1831, no. 8, pp. 1402-1411, 2013.

[48] K.-I. Okuhira, M. L. Fitzgerald, D. A. Sarracino et al., "Purification of ATP-binding cassette transporter A1 and associated binding proteins reveals the importance of $\beta 1$-syntrophin in cholesterol efflux," The Journal of Biological Chemistry, vol. 280, no. 47, pp. 39653-39664, 2005. 
[49] J. M. W. Taylor, F. Borthwick, C. Bartholomew, and A. Graham, "Overexpression of steroidogenic acute regulatory protein increases macrophage cholesterol efflux to apolipoprotein AI," Cardiovascular Research, vol. 86, no. 3, pp. 526-534, 2010.

[50] P. R. Manna, "Retinoid regulated macrophage cholesterol efflux involves the steroidogenic acute regulatory protein," Data in Brief, vol. 7, pp. 940-945, 2016.

[51] F. Borthwick, A.-M. Allen, J. M. Taylor, and A. Graham, “Overexpression of STARD3 in human monocyte/macrophages induces an anti-atherogenic lipid phenotype," Clinical Science, vol. 119, no. 7, pp. 265-272, 2010.

[52] J. M. W. Taylor, A.-M. Allen, and A. Graham, "Targeting mitochondrial $18 \mathrm{kDa}$ translocator protein (TSPO) regulates macrophage cholesterol efflux and lipid phenotype," Clinical Science, vol. 127, no. 10, pp. 603-613, 2014.

[53] J. E. Kanter, C. Tang, J. F. Oram, and K. E. Bornfeldt, "AcylCoA synthetase 1 is required for oleate and linoleate mediated inhibition of cholesterol efflux through ATP-binding cassette transporter A1 in macrophages," Biochimica et Biophysica Acta (BBA) - Molecular and Cell Biology of Lipids, vol. 1821, no. 3, pp. 358-364, 2012.

[54] J. Li and S. Zhang, "microRNA-150 inhibits the formation of macrophage foam cells through targeting adiponectin receptor 2," Biochemical and Biophysical Research Communications, vol. 476, no. 4, pp. 218-224, 2016.

[55] J. Y. Lee, J. Karwatsky, L. Ma, and X. Zha, "ABCA1 increases extracellular ATP to mediate cholesterol efflux to ApoA-I," American Journal of Physiology-Cell Physiology, vol. 301, no. 4, pp. C886-C894, 2011.

[56] M. Rana, A. Kumar, R. L. Tiwari et al., "IRAK regulates macrophage foam cell formation by modulating genes involved in cholesterol uptake and efflux," BioEssays, vol. 38, no. 7, pp. 591-604, 2016.

[57] T. Luo, J. Hu, D. Xi et al., "Lck inhibits heat shock protein 65mediated reverse cholesterol transport in T cells," The Journal of Immunology, vol. 197, no. 10, pp. 3861-3870, 2016.

[58] R. L. Kawashima and J. D. Medh, "Down-regulation of lipoprotein lipase increases ABCAl-mediated cholesterol efflux in THP-1 macrophages," Biochemical and Biophysical Research Communications, vol. 450, no. 4, pp. 1416-1421, 2014.

[59] D. Yan, M. I. Mäyränpää, J. Wong et al., “OSBP-related protein 8 (ORP8) suppresses ABCA1 expression and cholesterol efflux from macrophages," The Journal of Biological Chemistry, vol. 283, no. 1, pp. 332-340, 2008.

[60] G. Larigauderie, C. Furman, M. Jaye et al., "Adipophilin Enhances Lipid Accumulation and Prevents Lipid Efflux from THP-1 Macrophages: Potential Role in Atherogenesis," Arteriosclerosis, Thrombosis, and Vascular Biology, vol. 24, no. 3, pp. 504-510, 2004.

[61] W. Liu, J. Jiang, D. Yan et al., "Pentraxin 3 promotes oxLDL uptake and inhibits cholesterol efflux from macrophage-derived foam cells," Experimental and Molecular Pathology, vol. 96, no. 3, pp. 292-299, 2014.

[62] J. Zhang, J. A. Grieger, P. M. Kris-Etherton et al., "Walnut oil increases cholesterol efflux through inhibition of stearoyl CoA desaturase 1 in THP-1 macrophage-derived foam cells," Journal of Nutrition and Metabolism, vol. 8, 2011.

[63] B. P. Atshaves, O. Starodub, A. McIntosh et al., "Sterol carrier protein-2 alters high density lipoprotein-mediated cholesterol efflux," The Journal of Biological Chemistry, vol. 275, no. 47, pp. 36852-36861, 2000.
[64] N. Tamehiro, S. Zhou, K. Okuhira et al., "SPTLC1 binds ABCA1 to negatively regulate trafficking and cholesterol efflux activity of the transporter," Biochemistry, vol. 47, no. 23, pp. 6138-6147, 2008.

[65] L. Zeng, H. Liao, Y. Liu et al., "Sterol-responsive Elementbinding Protein (SREBP) 2 down-regulates ATP-binding cassette transporter A1 in vascular endothelial cells: A novel role of SREBP in regulating cholesterol metabolism," The Journal of Biological Chemistry, vol. 279, no. 47, pp. 48801-48807, 2004.

[66] J. E. McLaren, C. J. Calder, B. P. McSharry et al., "The TNFlike protein $1 \mathrm{~A}$-death receptor 3 pathway promotes macrophage foam cell formation in vitro," The Journal of Immunology, vol. 184, no. 10, pp. 5827-5834, 2010.

[67] M. Porsch-Özcürümez, T. Langmann, S. Heimerl et al., "The Zinc Finger Protein 202 (ZNF202) Is a Transcriptional Repressor of ATP Binding Cassette Transporter A1 (ABCA1) and ABCG1 Gene Expression and a Modulator of Cellular Lipid Efflux," The Journal of Biological Chemistry, vol. 276, no. 15, pp. 12427-12433, 2001.

[68] M. P. Adorni, F. Zimetti, J. T. Billheimer et al., "The roles of different pathways in the release of cholesterol from macrophages," Journal of Lipid Research, vol. 48, no. 11, pp. 2453-2462, 2007.

[69] X. Wang, H. L. Collins, M. Ranalletta et al., "Macrophage ABCA1 and ABCG1, but not SR-BI, promote macrophage reverse cholesterol transport in vivo," The Journal of Clinical Investigation, vol. 117, no. 8, pp. 2216-2224, 2007.

[70] M. Duong, H. L. Collins, W. Jin, I. Zanotti, E. Favari, and G. H. Rothblat, "Relative contributions of ABCA1 and SR-BI to cholesterol efflux to serum from fibroblasts and macrophages," Arteriosclerosis, Thrombosis, and Vascular Biology, vol. 26, no. 3, pp. 541-547, 2006.

[71] M. de la Llera-Moya, D. Drazul-Schrader, B. F. Asztalos, M. Cuchel, D. J. Rader, and G. H. Rothblat, "The ability to promote efflux via ABCA1 determines the capacity of serum specimens with similar high-density lipoprotein cholesterol to remove cholesterol from macrophages," Arteriosclerosis, Thrombosis, and Vascular Biology, vol. 30, no. 4, pp. 796-801, 2010.

[72] J. P. Slotte and E. L. Bierman, "Depletion of plasma-membrane sphingomyelin rapidly alters the distribution of cholesterol between plasma membranes and intracellular cholesterol pools in cultured fibroblasts," Biochemical Journal, vol. 250, no. 3, pp. 653-658, 1988.

[73] Y. Lange, J. Ye, and T. L. Steck, "How cholesterol is homeostasis is regulated by plasma membrane cholesterol in excess of phospholipids," Proceedings of the National Acadamy of Sciences of the United States of America, vol. 101, no. 32, pp. 11664-11667, 2004.

[74] N. A. Baumann, D. P. Sullivan, H. Ohvo-Rekilä et al., “Transport of newly synthesized sterol to the sterol-enriched plasma membrane occurs via nonvesicular equilibration," Biochemistry, vol. 44, no. 15, pp. 5816-5826, 2005.

[75] H. M. McConnell and M. Vrljic, "Liquid-liquid immiscibility in membranes," Annual Review of Biophysics, vol. 32, pp. 469-492, 2003.

[76] A. Das, J. L. Goldstein, D. D. Anderson, M. S. Brown, and A. Radhakrishnan, "Use of mutant 125I-Perfringolysin O to probe transport and organization of cholesterol in membranes of animal cells," Proceedings of the National Acadamy of Sciences of the United States of America, vol. 110, no. 26, pp. 10580-10585, 2013.

[77] J. Iqbal and M. M. Hussain, "Intestinal lipid absorption," American Journal of Physiology-Endocrinology and Metabolism, vol. 296, no. 6, pp. E1183-E1194, 2009. 
[78] J. M. Dietschy, S. D. Turley, and D. K. Spady, "Role of liver in the maintenance of cholesterol and low density lipoprotein homeostasis in different animal species, including humans," Journal of Lipid Research, vol. 34, no. 10, pp. 1637-1659, 1993.

[79] M. Y. M. Van Der Wulp, H. J. Verkade, and A. K. Groen, "Regulation of cholesterol homeostasis," Molecular and Cellular Endocrinology, vol. 368, no. 1-2, pp. 1-16, 2013.

[80] A. Nohturfft and C. Z. Shao, "Coordination of lipid metabolism in membrane biogenesis," Annual Review of Cell and Developmental Biology, vol. 25, pp. 539-566, 2009.

[81] E. Ikonen, "Cellular cholesterol trafficking and compartmentalization," Nature Reviews Molecular Cell Biology, vol. 9, no. 2, pp. 125-138, 2008.

[82] J. Luo, L. Jiang, H. Yang, and B.-L. Song, "Routes and mechanisms of post-endosomal cholesterol trafficking: A story that never ends," Traffic, vol. 18, no. 4, pp. 209-217, 2017.

[83] D. Cheng, C. C. Y. Chang, X.-M. Qu, and T.-Y. Chang, "Activation of acyl-coenzyme A:cholesterol acyltransferase by cholesterol or by oxysterol in a cell-free system," The Journal of Biological Chemistry, vol. 270, no. 2, pp. 685-695, 1995.

[84] F. Wilfling, J. T. Haas, T. C. Walther, and R. V. F. Jr, "Lipid droplet biogenesis," Current Opinion in Cell Biology, vol. 29, no. 1, pp. 39-45, 2014.

[85] H. F. Hashemi and J. M. Goodman, "The life cycle of lipid droplets," Current Opinion in Cell Biology, vol. 33, pp. 119-124, 2015.

[86] A. D. Barbosa and S. Siniossoglou, "Function of lipid dropletorganelle interactions in lipid homeostasis," Biochimica et Biophysica Acta (BBA) - Molecular Cell Research, vol. 1864, no. 9, pp. 1459-1468, 2017.

[87] M. Ouimet and Y. L. Marcel, "Regulation of lipid droplet cholesterol efflux from macrophage foam cells," Arteriosclerosis, Thrombosis, and Vascular Biology, vol. 32, no. 3, pp. 575-581, 2012.

[88] M. Ouimet, V. Franklin, E. Mak, X. Liao, I. Tabas, and Y. L. Marcel, "Autophagy regulates cholesterol efflux from macrophage foam cells via lysosomal acid lipase," Cell Metabolism, vol. 13, no. 6, pp. 655-667, 2011.

[89] S.-J. Jeong, M.-N. Lee, and G. T. Oh, "The role of macrophage lipophagy in reverse cholesterol transport," Endocrinology and Metabolism Clinics of North America, vol. 32, no. 1, pp. 41-46, 2017.

[90] Y. Yamauchi, S. Yokoyama, and T.-Y. Chang, "ABCA1dependent sterol release: Sterol molecule specifi city and potential membrane domain for HDL biogenesis," Journal of Lipid Research, vol. 57, no. 1, pp. 77-88, 2016.

[91] A. J. Mendez, "Cholesterol efflux mediated by apolipoproteins is an active cellular process distinct from efflux mediated by passive diffusion," Journal of Lipid Research, vol. 38, no. 9, pp. 1807-1821, 1997.

[92] G. Van Meer, "Lipid traffic in animal cells," Annual Review of Cell and Developmental Biology, vol. 5, pp. 247-275, 1989.

[93] A. D. Gillon, C. F. Latham, and E. A. Miller, "Vesicle-mediated ER export of proteins and lipids," Biochimica et Biophysica Acta (BBA) - Molecular and Cell Biology of Lipids, vol. 1821, no. 8, pp. 1040-1049, 2012.

[94] R. E. Soccio and J. L. Breslow, "Intracellular cholesterol transport," Arteriosclerosis, Thrombosis, and Vascular Biology, vol. 24, no. 7, pp. 1150-1160, 2004.

[95] M. R. Kaplan and R. D. Simoni, "Transport of cholesterol from the endoplasmic reticulum to the plasma membrane," The Journal of Cell Biology, vol. 101, no. 2, pp. 446-453, 1985.
[96] S. Heino, S. Lusa, P. Somerharju, C. Ehnholm, V. M. Olkkonen, and E. Ikonen, "Dissecting the role of the Golgi complex and lipid rafts in biosynthetic transport of cholesterol to the cell surface," Proceedings of the National Acadamy of Sciences of the United States of America, vol. 97, no. 15, pp. 8375-8380, 2000.

[97] R. F. DeGrella and R. D. Simoni, "Intracellular transport of cholesterol to the plasma membrane," The Journal of Biological Chemistry, vol. 257, no. 23, pp. 14256-14262, 1982.

[98] D. B. Iaea, S. Mao, F. W. Lund, and F. R. Maxfield, "Role of STARD4 in sterol transport between the endocytic recycling compartment and the plasma membrane," Molecular Biology of the Cell (MBoC), vol. 28, no. 8, pp. 1111-1122, 2017.

[99] Y. Li and W. A. Prinz, "ATP-binding cassette (ABC) transporters mediate nonvesicular, raft-modulated sterol movement from the plasma membrane to the endoplasmic reticulum," The Journal of Biological Chemistry, vol. 279, no. 43, pp. 4522645234, 2004.

[100] S. Raychaudhuri and W. A. Prinz, "The diverse functions of oxysterol-binding proteins," Annual Review of Cell and Developmental Biology, vol. 26, pp. 157-177, 2010.

[101] G. Drin, J. Moser Von Filseck, and A. Čopič, "New molecular mechanisms of inter-organelle lipid transport," Biochemical Society Transactions, vol. 44, no. 2, pp. 486-492, 2016.

[102] V. M. Olkkonen, "OSBP-related protein family in lipid transport over membrane contact sites," Lipid Insights, vol. 2015, pp. 1-9, 2015.

[103] T. Daniele and M. V. Schiaffino, "Organelle biogenesis and interorganellar connections: Better in contact than in isolation," Communicative and Integrative Biology, vol. 7, no. 4, pp. e29587e29587-6, 2014.

[104] E. Quon and C. T. Beh, "Membrane contact sites: Complex zones for membrane association and lipid Exchange," Lipid Insights, vol. 2015, pp. 55-63, 2015.

[105] F. Schroeder, B. P. Atshaves, A. L. McIntosh et al., "Sterol carrier protein-2: New roles in regulating lipid rafts and signaling," Biochimica et Biophysica Acta (BBA) - Molecular and Cell Biology of Lipids, vol. 1771, no. 6, pp. 700-718, 2007.

[106] B. Mesmin, J. Bigay, J. Moser Von Filseck, S. Lacas-Gervais, G. Drin, and B. Antonny, "XA four-step cycle driven by PI(4)P hydrolysis directs sterol/PI(4)P exchange by the ER-Golgi Tether OSBP," Cell, vol. 155, no. 4, pp. X830-843, 2013.

[107] F. R. Taylor, S. E. Saucier, E. P. Shown, E. J. Parish, and A. A. Kandutsch, "Correlation between oxysterol binding to a cytosolic binding protein and potency in the repression of hydroxymethylglutaryl coenzyme A reductase," The Journal of Biological Chemistry, vol. 259, no. 20, pp. 12382-12387, 1984.

[108] I. P. Artemenko, D. Zhao, D. B. Hales, K. H. Hales, and C. R. Jefcoate, "Mitochondrial Processing of Newly Synthesized Steroidogenic Acute Regulatory Protein (StAR), but Not Total StAR, Mediates Cholesterol Transfer to Cytochrome P450 Side Chain Cleavage Enzyme in Adrenal Cells," The Journal of Biological Chemistry, vol. 276, no. 49, pp. 46583-46596, 2001.

[109] L. P. Wilhelm, C. Wendling, B. Védie et al., "STARD3 mediates endoplasmic reticulum-to-endosome cholesterol transport at membrane contact sites," EMBO Journal, vol. 36, no. 10, pp. 1412-1433, 2017.

[110] B. Mesmin, N. H. Pipalia, F. W. Lund et al., "STARD4 abundance regulates sterol transport and sensing," Molecular Biology of the Cell (MBoC), vol. 22, no. 21, pp. 4004-4015, 2011.

[111] J. Garbarino, M. Pan, H. F. Chin, F. W. Lund, F. R. Maxfield, and J. L. Breslow, "STARD4 knockdown in HepG2 cells disrupts 
cholesterol trafficking associated with the plasma membrane, ER, and ERC," Journal of Lipid Research, vol. 53, no. 12, pp. 27162725, 2012.

[112] L. Puglielli, A. Rigotti, A. V. Greco, M. J. Santos, and F. Nervi, "Sterol carrier protein-2 is involved in cholesterol transfer from the endoplasmic reticulum to the plasma membrane in human fibroblasts," The Journal of Biological Chemistry, vol. 270, no. 32, pp. 18723-18726, 1995.

[113] C. L. Baum, E. J. Reschly, A. K. Gayen, M. E. Groh, and K. Schadick, "Sterol carrier protein-2 overexpression enhances sterol cycling and inhibits cholesterol ester synthesis and high density lipoprotein cholesterol secretion," The Journal of Biological Chemistry, vol. 272, no. 10, pp. 6490-6498, 1997.

[114] S. M. Storey, B. P. Atshaves, A. L. McIntosh et al., "Effect of sterol carrier protein-2 gene ablation on HDL-mediated cholesterol efflux from cultured primary mouse hepatocytes," American Journal of Physiology-Gastrointestinal and Liver Physiology, vol. 299, no. 1, pp. G244-G254, 2010.

[115] S. M. Storey, A. L. McIntosh, H. Huang et al., "Intracellular cholesterol-binding proteins enhance HDL-mediated cholesterol uptake in cultured primary mouse hepatocytes," American Journal of Physiology-Gastrointestinal and Liver Physiology, vol. 302, no. 8, pp. G824-G839, 2012.

[116] P. E. Fielding and C. J. Fielding, "Plasma Membrane Caveolae Mediate the Efflux of Cellular Free Cholesterol," Biochemistry, vol. 34, no. 44, pp. 14288-14292, 1995.

[117] E. J. Smart, Y.-S. Ying, W. C. Donzell, and R. G. W. Anderson, “A role for caveolin in transport of cholesterol from endoplasmic reticulum to plasma membrane," The Journal of Biological Chemistry, vol. 271, no. 46, pp. 29427-29435, 1996.

[118] M. G. Sorci-Thomas, J. S. Owen, B. Fulp et al., "Nascent high density lipoproteins formed by ABCA1 resemble lipid rafts and are structurally organized by three apoA-I monomers," Journal of Lipid Research, vol. 53, no. 9, pp. 1890-1909, 2012.

[119] A. R. Busija, H. H. Patel, and P. A. Insel, "Caveolins and cavins in the trafficking, maturation, and degradation of caveolae: Implications for cell physiology," American Journal of Physiology-Cell Physiology, vol. 312, no. 4, pp. C459-C477, 2017.

[120] M. Murata, J. Peränen, R. Schreiner, F. Wieland, T. V. Kurzchalia, and K. Simons, "VIP21/caveolin is a cholesterol-binding protein," Proceedings of the National Acadamy of Sciences of the United States of America, vol. 92, no. 22, pp. 10339-10343, 1995.

[121] M. Zhou, R. D. Parr, A. D. Petrescu et al., "Sterol carrier protein-2 directly interacts with caveolin-1 in vitro and in vivo," Biochemistry, vol. 43, no. 23, pp. 7288-7306, 2004.

[122] J. M. R. Patlolla, M. V. Swamy, J. Raju, and C. V. Rao, “Overexpression of caveolin-1 in experimental colon adenocarcinomas and human colon cancer cell lines," Oncology Reports, vol. 11, no. 5, pp. 957-963, 2004.

[123] A. Pol, R. Luetterforst, M. Lindsay, S. Heino, E. Ikonen, and R. G. Parton, "A caveolin dominant negative mutant associates with lipid bodies and induces intracellular cholesterol imbalance," The Journal of Cell Biology, vol. 152, no. 5, pp. 1057-1070, 2001.

[124] S. Le Lay, M. Rodriguez, W. Jessup et al., "Caveolin-1-mediated apolipoprotein A-I membrane binding sites are not required for cholesterol efflux," PLoS ONE, vol. 6, no. 8, Article ID e23353, 2011.

[125] M. C. Phillips, "Molecular mechanisms of cellular cholesterol efflux," The Journal of Biological Chemistry, vol. 289, no. 35, pp. 24020-24029, 2014.

[126] S. Yokoyama, "Release of cellular cholesterol: Molecular mechanism for cholesterol homeostasis in cells and in the body,"
Biochimica et Biophysica Acta (BBA) - Molecular and Cell Biology of Lipids, vol. 1529, no. 1-3, pp. 231-244, 2000.

[127] L. R. McLean and M. C. Phillips, "Mechanism of Cholesterol and Phosphatidylcholine Exchange or Transfer between Unilamellar Vesicles," Biochemistry, vol. 20, no. 10, pp. 2893-2900, 1981.

[128] J. D. Jones and T. E. Thompson, "Spontaneous Phosphatidylcholine Transfer by Collision between Vesicles at High Lipid Concentration," Biochemistry, vol. 28, no. 1, pp. 129-134, 1989.

[129] T. L. Steck, F. J. Kezdy, and Y. Lange, "An activation-collision mechanism for cholesterol transfer between membranes," The Journal of Biological Chemistry, vol. 263, no. 26, pp. 13023-13031, 1988.

[130] M. H. Gottlieb, "Rates of cholesterol exchange between human erythrocytes and plasma lipoproteins," Biochimica et Biophysica Acta, vol. 600, no. 2, pp. 530-541, 1980.

[131] J. D. Toledo, M. A. Tricerri, B. Córsico, and H. A. Garda, "Cholesterol flux between lipid vesicles and apolipoprotein AI discs of variable size and composition," Archives of Biochemistry and Biophysics, vol. 380, no. 1, pp. 63-70, 2000.

[132] N. Matsuzaki, T. Handa, and M. Nakano, "Kinetic and Thermodynamic Analysis of Cholesterol Transfer between Phospholipid Vesicles and Nanodiscs," The Journal of Physical Chemistry B, vol. 119, no. 30, pp. 9764-9771, 2015.

[133] A. D. Dergunov, J. Taveirne, B. Vanloo, H. Caster, and M. Rosseneu, "Structural organization of lipid phase and proteinlipid interface in apolipoprotein-phospholipid recombinants: Influence of cholesterol," Biochimica et Biophysica Acta (BBA) - Lipids and Lipid Metabolism, vol. 1346, no. 2, pp. 131-146, 1997.

[134] E. B. Neufeld, A. T. Remaley, S. J. Demosky et al., "Cellular Localization and Trafficking of the Human ABCA1 Transporter," The Journal of Biological Chemistry, vol. 276, no. 29, pp. 27584-27590, 2001.

[135] E. B. Neufeld, J. A. Stonik, S. J. Demosky Jr. et al., “The ABCA1 transporter modulates late endocytic trafficking: insights from the correction of the genetic defect in Tangier disease," The Journal of Biological Chemistry, vol. 279, no. 15, pp. 15571-15578, 2004.

[136] E. Boadu, R. C. Nelson, and G. A. Francis, "ABCA1-dependent mobilization of lysosomal cholesterol requires functional Niemann-Pick C2 but not Niemann-Pick C1 protein," Biochimica et Biophysica Acta (BBA) - Molecular and Cell Biology of Lipids, vol. 1821, no. 3, pp. 396-404, 2012.

[137] Y. Yamauchi, N. Iwamoto, M. A. Rogers et al., "Deficiency in the lipid exporter ABCA1 impairs retrograde sterol movement and disrupts sterol sensing at the endoplasmic reticulum," The Journal of Biological Chemistry, vol. 290, no. 39, pp. 2346423477, 2015.

[138] K. Takahashi, Y. Kimura, K. Nagata, A. Yamamoto, M. Matsuo, and K. Ueda, "ABC proteins: Key molecules for lipid homeostasis," Medical Molecular Morphology, vol. 38, no. 1, pp. 2-12, 2005.

[139] F. Quazi and R. S. Molday, "Differential phospholipid substrates and directional transport by ATP binding cassette proteins ABCA1, ABCA7, and ABCA4 and disease-causing mutants," The Journal of Biological Chemistry, vol. 288, no. 48, pp. 3441434426, 2013.

[140] V. Hsieh, M.-J. Kim, I. C. Gelissen et al., "Cellular cholesterol regulates ubiquitination and degradation of the cholesterol export proteins ABCA1 and ABCG1," The Journal of Biological Chemistry, vol. 289, no. 11, pp. 7524-7536, 2014.

[141] R. R. Singaraja, M. H. Kang, K. Vaid et al., "Palmitoylation of ATP-binding cassette transporter Al Is essential for its 
trafficking and function," Circulation Research, vol. 105, no. 2, pp. 138-147, 2009.

[142] P. T. Duong, H. L. Collins, M. Nickel, S. Lund-Katz, G. H. Rothblat, and M. C. Phillips, "Characterization of nascent HDL particles and microparticles formed by ABCA1-mediated efflux of cellular lipids to apoA-I," Journal of Lipid Research, vol. 47, no. 4, pp. 832-843, 2006.

[143] K. L. Gillotte, W. S. Davidson, S. Lund-Katz, G. H. Rothblat, and M. C. Phillips, "Removal of cellular cholesterol by pre- $\beta$ HDL involves plasma membrane microsolubilization," Journal of Lipid Research, vol. 39, no. 10, pp. 1918-1928, 1998.

[144] W. Möbius, E. van Donselaar, Y. Ohno-Iwashita et al., "Recycling compartments and the internal vesicles of multivesicular bodies harbor most of the cholesterol found in the endocytic pathway," Traffic, vol. 4, no. 4, pp. 222-231, 2003.

[145] M. J. Van Dam, E. De Groot, S. M. Clee et al., "Association between increased arterial-wall thickness and impairment in ABCA1-driven cholesterol efflux: An observational study," The Lancet, vol. 359, no. 9300, pp. 37-41, 2002.

[146] A. E. Bochem, D. F. Van Wijk, A. E. Holleboom et al., "ABCA1 mutation carriers with low high-density lipoprotein cholesterol are characterized by a larger atherosclerotic burden," European Heart Journal, vol. 34, no. 4, pp. 286-291, 2013.

[147] R. Frikke-Schmidt, B. G. Nordestgaard, M. C. A. Stene et al., "Association of loss-of-function mutations in the ABCA1 gene with high-density lipoprotein cholesterol levels and risk of ischemic heart disease," Journal of the American Medical Association, vol. 299, no. 21, pp. 2524-2532, 2008.

[148] D. Karunakaran, A. B. Thrush, and M. A. Nguyen, "Macrophage mitochondrial energy status regulates cholesterol efflux and is enhanced by anti-miR33 in atherosclerosis," Circulation Research, vol. 117, no. 3, pp. 266-278, 2015.

[149] Y. M. Morizawa, Y. Hirayama, N. Ohno et al., "Reactive astrocytes function as phagocytes after brain ischemia via ABCA1mediated pathway," Nature Communications, vol. 8, no. 1, article no. 28, 2017.

[150] A. Chroni, T. Liu, M. L. Fitzgerald, M. W. Freeman, and V. I. Zannis, "Cross-Linking and Lipid Efflux Properties of ApoAI Mutants Suggest Direct Association between ApoA-I Helices and ABCA1," Biochemistry, vol. 43, no. 7, pp. 2126-2139, 2004.

[151] M. L. Fitzgerald, A. L. Morris, A. Chroni, A. J. Mendez, V. I. Zannis, and M. W. Freeman, "ABCA1 and amphipathic apolipoproteins form high-affinity molecular complexes required for cholesterol efflux," Journal of Lipid Research, vol. 45, no. 2, pp. 287-294, 2004.

[152] C. Vedhachalam, A. B. Ghering, W. S. Davidson, S. Lund-Katz, G. H. Rothblat, and M. C. Phillips, "ABCA1-induced cell surface binding sites for ApoA-I," Arteriosclerosis, Thrombosis, and Vascular Biology, vol. 27, no. 7, pp. 1603-1609, 2007.

[153] G. Datta, M. Chaddha, S. Hama et al., "Effects of increasing hydrophobicity on the physical-chemical and biological properties of a class A amphipathic helical peptide," Journal of Lipid Research, vol. 42, no. 7, pp. 1096-1104, 2001.

[154] J. F. Nunez and J. B. Swaney, "Interaction between hepatic microsomal membrane lipids and apolipoprotein A-I," The Journal of Biological Chemistry, vol. 259, no. 14, pp. 9141-9148, 1984.

[155] Y. D. Landry, M. Denis, S. Nandi, S. Bell, A. M. Vaughan, and X. Zha, "ATP-binding cassette transporter Al expression disrupts raft membrane microdomains through its ATPase-related functions," The Journal of Biological Chemistry, vol. 281, no. 47, pp. 36091-36101, 2006.
[156] K. O. Nagata, C. Nakada, R. S. Kasai, A. Kusumi, and K. Ueda, "ABCA1 dimer-monomer interconversion during HDL generation revealed by single-molecule imaging," Proceedings of the National Acadamy of Sciences of the United States of America, vol. 110, no. 13, pp. 5034-5039, 2013.

[157] H. H. Hassan, M. Denis, D.-Y. D. Lee et al., "Identification of an ABCA1-dependent phospholipid-rich plasma membrane apolipoprotein A-I binding site for nascent HDL formation: Implications for current models of HDL biogenesis," Journal of Lipid Research, vol. 48, no. 11, pp. 2428-2442, 2007.

[158] S. Wang, K. Gulshan, G. Brubaker, S. L. Hazen, and J. D. Smith, "ABCA1 mediates unfolding of apolipoprotein AI N terminus on the cell surface before lipidation and release of nascent highdensity lipoprotein," Arteriosclerosis, Thrombosis, and Vascular Biology, vol. 33, no. 6, pp. 1197-1205, 2013.

[159] E. Pandzic, I. C. Gelissen, R. Whan et al., "The ATP binding cassette transporter, ABCG1, localizes to cortical actin filaments," Scientific Reports, vol. 7, 2017.

[160] E. B. Neufeld, K. O’Brien, A. D. Walts et al., "Cellular localization and trafficking of the human abcgl transporter," Biology, vol. 3, no. 4, pp. 781-800, 2014.

[161] E. J. Tarling and P. A. Edwards, "ATP binding cassette transporter G1 (ABCG1) is an intracellular sterol transporter," Proceedings of the National Acadamy of Sciences of the United States of America, vol. 108, no. 49, pp. 19719-19724, 2011.

[162] E. J. Tarling and P. A. Edwards, "Intracellular Localization of Endogenous Mouse ABCG1 Is Mimicked by Both ABCG1-L550 and ABCG1-P550-Brief Report," Arteriosclerosis, Thrombosis, and Vascular Biology, vol. 36, no. 7, pp. 1323-1327, 2016.

[163] I. D. Kerr, A. J. Haider, and I. C. Gelissen, "The ABCG family of membrane-associated transporters: You don't have to be big to be mighty," British Journal of Pharmacology, vol. 164, no. 7, pp. 1767-1779, 2011.

[164] H.-M. Gu, G. Li, X. Gao, L. G. Berthiaume, and D.-W. Zhang, "Characterization of palmitoylation of ATP binding cassette transporter G1: Effect on protein trafficking and function," Biochimica et Biophysica Acta (BBA) - Molecular and Cell Biology of Lipids, vol. 1831, no. 6, pp. 1067-1078, 2013.

[165] L. J. Sharpe, G. Rao, P. M. Jones et al., "Cholesterol sensing by the ABCG1 lipid transporter: Requirement of a CRAC motif in the final transmembrane domain," Biochimica et Biophysica Acta (BBA) - Molecular and Cell Biology of Lipids, vol. 1851, no. 7, Article ID 57777, pp. 956-964, 2015.

[166] H. Hirayama, Y. Kimura, N. Kioka, M. Matsuo, and K. Ueda, "ATPase activity of human ABCG1 is stimulated by cholesterol and sphingomyelin," Journal of Lipid Research, vol. 54, no. 2, pp. 496-502, 2013

[167] S. Sankaramarayanan, J. F. Oram, B. F. Asztalos et al., "Effects of acceptor composition and mechanism of ABCG1-mediated cellular free cholesterol efflux," Journal of Lipid Research, vol. 50, no. 2, pp. 275-284, 2009.

[168] E. B. Neufeld, K. O’Brien, A. D. Walts et al., "The human ABCG1 transporter mobilizes plasma membrane and late endosomal non-sphingomyelin-associated-cholesterol for efflux and esterification," Biology, vol. 3, no. 4, pp. 866-891, 2014.

[169] M. A. Kennedy, G. C. Barrera, K. Nakamura et al., "ABCG1 has a critical role in mediating cholesterol efflux to HDL and preventing cellular lipid accumulation," Cell Metabolism, vol. 1, no. 2, pp. 121-131, 2005.

[170] W. Le Goff and G. M. Dallinga-Thie, "ABCG1: Not as good as expected?” Atherosclerosis, vol. 219, no. 2, pp. 393-394, 2011. 
[171] L. Hardy, E. Frisdal, and W. Le Goff, "Critical Role of the Human ATP-Binding Cassette G1 Transporter in Cardiometabolic Diseases," International Journal of Molecular Sciences, vol. 18, no. 9, p. 1892, 2017.

[172] N. Terasaka, M. Westerterp, J. Koetsveld et al., "ATP-binding cassette transporter G1 and high-density lipoprotein promote endothelial NO synthesis through a decrease in the interaction of caveolin-1 and endothelial NO synthase," Arteriosclerosis, Thrombosis, and Vascular Biology, vol. 30, no. 11, pp. 2219-2225, 2010.

[173] M. L. Varban, F. Rinninger, N. Wang et al., "Targeted mutation reveals a central role for SR-BI in hepatic selective uptake of high density lipoprotein cholesterol," Proceedings of the National Acadamy of Sciences of the United States of America, vol. 95, no. 8, pp. 4619-4624, 1998.

[174] K. T. Landschulz, R. K. Pathak, A. Rigotti, M. Krieger, and H. H. Hobbs, "Regulation of scavenger receptor, class B, type I, a high density lipoprotein receptor, in liver and steroidogenic tissues of the rat," The Journal of Clinical Investigation, vol. 98, no. 4, pp. 984-995, 1996.

[175] B. L. Trigatti, "SR-B1 and PDZK1: Partners in HDL regulation," Current Opinion in Lipidology, vol. 28, no. 2, pp. 201-208, 2017.

[176] O. Kocher and M. Krieger, "Role of the adaptor protein PDZK1 in controlling the HDL receptor SR-BI," Current Opinion in Lipidology, vol. 20, no. 3, pp. 236-241, 2009.

[177] R. Riessen, D. I. Axel, M. Fenchel, U. U. Herzog, H. Roßmann, and K. R. Karsch, "Effect of HMG-CoA reductase inhibitors on extracellular matrix expression in human vascular smooth muscle cells," Basic Research in Cardiology, vol. 94, no. 5, pp. 322-332, 1999.

[178] J. Babitt, B. Trigatti, A. Rigotti et al., "Murine SR-BI, a high density lipoprotein receptor that mediates selective lipid uptake, is $\mathrm{N}$-glycosylated and fatty acylated and colocalizes with plasma membrane caveolae," The Journal of Biological Chemistry, vol. 272, no. 20, pp. 13242-13249, 1997.

[179] M. Ikemoto, H. Arai, D. Feng et al., "Identification of a PDZdomain-containing protein that interacts with the scavenger receptor class B type I," Proceedings of the National Acadamy of Sciences of the United States of America, vol. 97, no. 12, pp. 65386543, 2000.

[180] S. Acton, A. Rigotti, K. T. Landschulz, S. Xu, H. H. Hobbs, and M. Krieger, "Identification of scavenger receptor SR-BI as high density lipoprotein receptor," Science, vol. 271, no. 5248, pp. 518520, 1996.

[181] A. Boullier, D. A. Bird, M.-K. Chang et al., "Scavenger receptors, oxidized LDL, and atherosclerosis," Annals of the New York Academy of Sciences, vol. 947, pp. 214-223, 2001.

[182] S. T. Thuahnai, S. Lund-Katz, P. Dhanasekaran et al., "Scavenger receptor class B type I-mediated cholesteryl ester-selective uptake and efflux of unesterified cholesterol: Influence of high density lipoprotein size and structure," The Journal of Biological Chemistry, vol. 279, no. 13, pp. 12448-12455, 2004.

[183] W. V. Rodrigueza, S. T. Thuahnai, R. E. Temel, S. Lund-Katz, M. C. Phillips, and D. L. Williams, "Mechanism of scavenger receptor class B type I-mediated selective uptake of cholesteryl esters from high density lipoprotein to adrenal cells," The Journal of Biological Chemistry, vol. 274, no. 29, pp. 2034420350, 1999.

[184] M. De La Llera-Moya, G. H. Rothblat, M. A. Connelly et al., "Scavenger receptor BI (SR-BI) mediates free cholesterol flux independently of HDL tethering to the cell surface," Journal of Lipid Research, vol. 40, no. 3, pp. 575-580, 1999.
[185] G. A. Kartz, R. L. Holme, K. Nicholson, and D. Sahoo, "SR$\mathrm{BI} / \mathrm{CD} 36$ chimeric receptors define extracellular subdomains of SR-BI critical for cholesterol transport," Biochemistry, vol. 53, no. 39, pp. 6173-6182, 2014.

[186] M. Yu, T. Y. Lau, S. A. Carr, and M. Krieger, "Contributions of a disulfide bond and a reduced cysteine side chain to the intrinsic activity of the high-density lipoprotein receptor SRBI," Biochemistry, vol. 51, no. 50, pp. 10044-10055, 2012.

[187] R. L. Holme, J. J. Miller, K. Nicholson, and D. Sahoo, “Tryptophan 415 Is Critical for the Cholesterol Transport Functions of Scavenger Receptor BI," Biochemistry, vol. 55, no. 1, pp. 103-113, 2016.

[188] A. C. Chadwick and D. Sahoo, "Functional Characterization of Newly-Discovered Mutations in Human SR-BI," PLoS ONE, vol. 7, no. 9, Article ID e45660, 2012. 


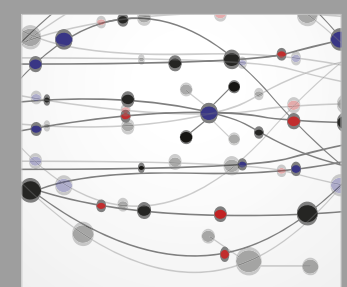

The Scientific World Journal
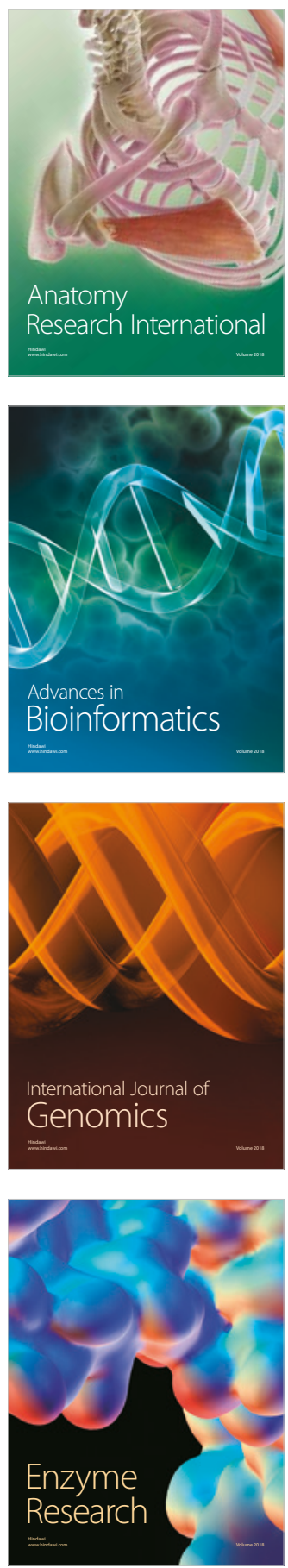
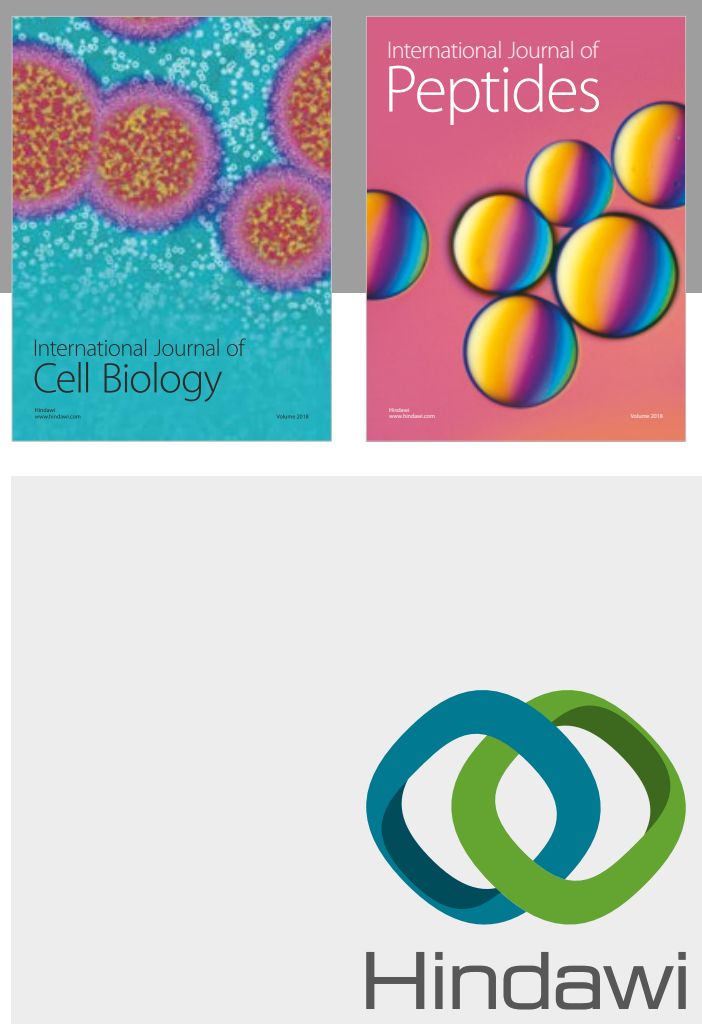

Submit your manuscripts at

www.hindawi.com
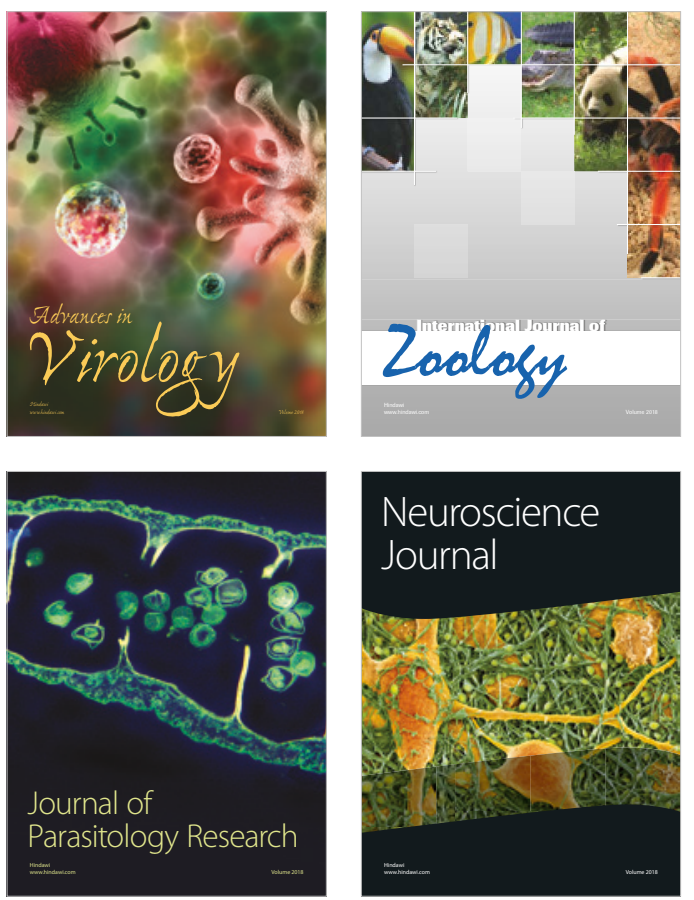
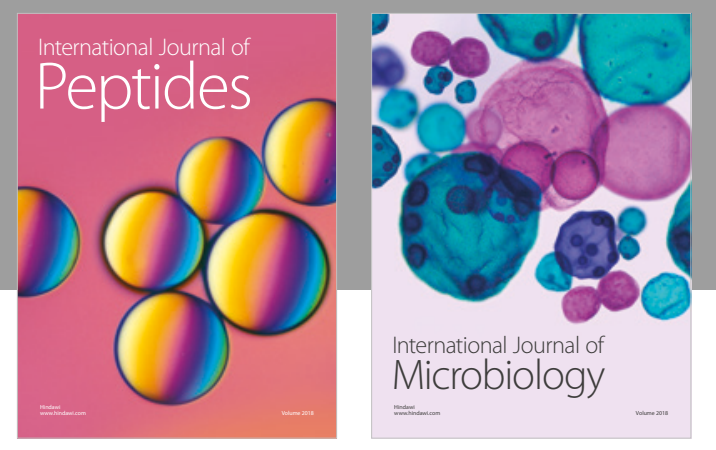

nternational Journal of Microbiology
Journal of
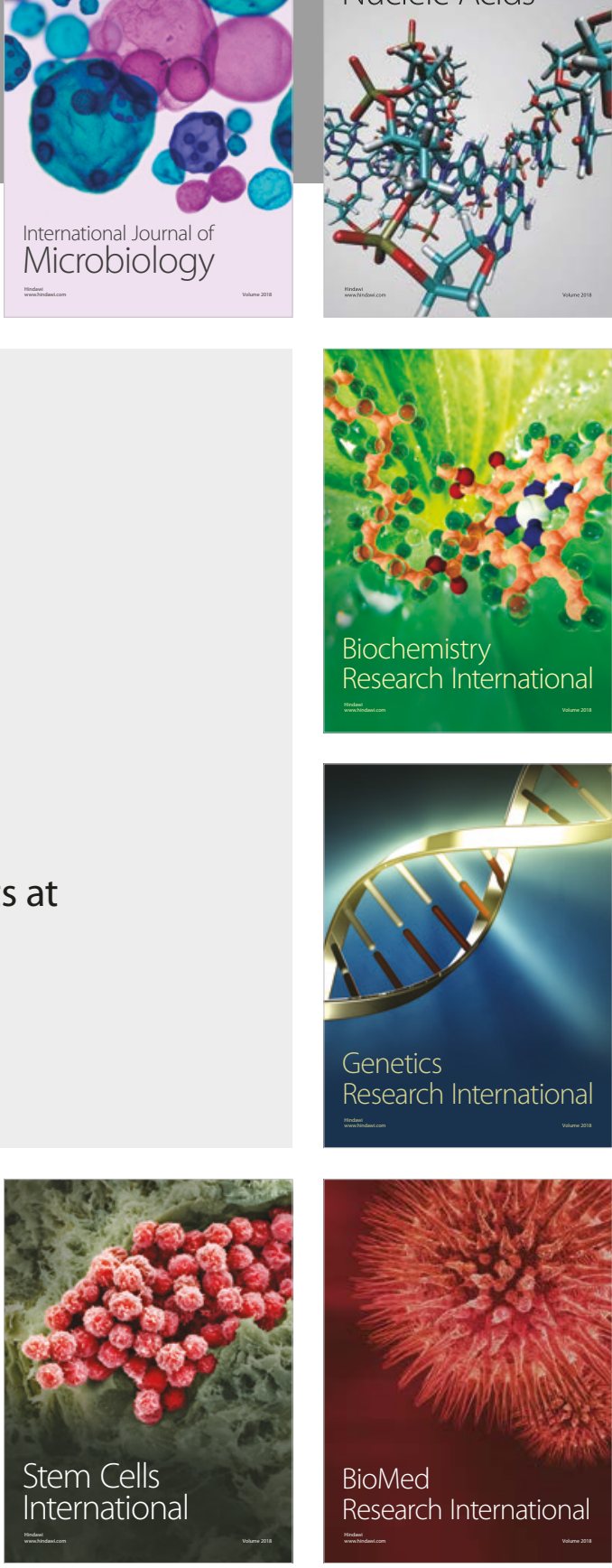
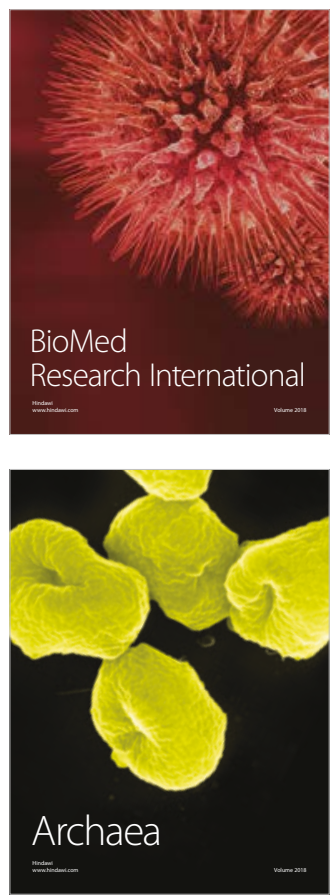